\title{
On the optimal relative orientation of radicals in the cryptochrome magnetic
} compass

\author{
Chadsley Atkins, ${ }^{\dagger, 1}$ Kieran Bajpai, $^{\dagger, 1}$ Jeremy Rumball, ${ }^{\dagger, 1}$ and Daniel R. Kattnig ${ }^{*, 1,2}$ \\ ${ }^{1}$ Institute and Department of Physics, University of Exeter, North Park Road, Exeter EX4 4QL, UK \\ ${ }^{2}$ Living Systems Institute and Department of Physics, University of Exeter, Stocker Road, Exeter EX4 \\ 4QD, UK \\ * d.r.kattnig@exeter.ac.uk \\ † These authors contributed equally to this work.
}

Abstract

Birds appear to be equipped with an innate magnetic compass. One biophysical model of this sense relies on spin dynamics in photo-generated radical pairs in the protein cryptochrome. This study employs a systematic approach to predict the dependence of the compass sensitivity on the relative orientation of the constituent radicals for spin systems comprising up to 21 hyperfine interactions. Evaluating measures of compass sensitivity (anisotropy) and precision (optimality) derived from the singlet yield, we find the ideal relative orientations for the radical pairs consisting of the flavin anion $\left(\mathrm{F}^{\bullet-}\right)$ coupled with a tryptophan cation $\left(\mathrm{W}^{\bullet+}\right)$ or tyrosine radical $\left(\mathrm{Y}^{\bullet}\right)$. For the geomagnetic field, the two measures are found to be anti-correlated in $\left[\mathrm{F}^{\bullet-} \mathrm{W}^{\bullet+}\right]$. The angle spanned by the normals to the aromatic planes of the radicals is the decisive parameter determining the compass sensitivity. The third tryptophan of the tryptophan triad/tetrad, which has been implicated with magnetosensitive responses, exhibits a comparably large anisotropy, but unfavorable optimality. Its anisotropy could be boosted by an additional $\sim 50 \%$ by optimizing the relative orientation of the radicals. For a coherent lifetime of $1 \mu \mathrm{s}$, the maximal relative anisotropy of $\left[\mathrm{F}^{\bullet-} \mathrm{W}^{\bullet+}\right]$ is $0.27 \%$. [ $\left.\mathrm{F}^{\bullet-} \mathrm{Y}^{\bullet}\right]$ radical pairs outperform $\left[\mathrm{F}^{\bullet-} \mathrm{W}^{\bullet+}\right]$ for most relative orientations. Furthermore, anisotropy and optimality can be simultaneously maximized. The entanglement decays rapidly, implicating it as a situational byproduct rather than a fundamental driver within the avian compass. In magnetic fields of higher intensity, the relative orientation of radicals in $\left[\mathrm{F}^{\bullet-} \mathrm{W}^{\bullet+}\right]$ is less important than for the geomagnetic field.

\section{Introduction}

Several organisms appear to perceive the Earth's weak magnetic field, for orientation and navigational purposes [1-3]. In most instances, the mechanistic basis of this sense remains ambiguous. Spin dynamics in spin-sensitive radical pair reactions are considered to be responsible for some of the magnetoreceptive traits $[4,5]$. Others are discussed to rely on a classical mechanism based on ferromagnetic or superparamagnetic particles from, e.g., magnetite [1-3]. A magnetic compass in migratory songbirds is hypothesized to rely on photo-induced radical pairs generated within the flavoprotein cryptochrome, located within the animal's retinae $[4,6]$. A similar mechanism is thought to apply to certain magnetosensitive phenotypes in plants [7-10], fruit flies [11], cockroaches [12] and newts [13]. Convincing support for this hypothesis includes the experimental demonstration that the photo-reduction of isolated cryptochromes from $A$. thaliana and $D$. melanogaster can be influenced by static magnetic fields, albeit at field strengths exceeding the geomagnetic field ( $50 \mu \mathrm{T})$ [14-17]. The effect has been attributed to the Radical Pair Mechanism (RPM), which relates reaction outcomes 
to hyperfine-driven spin dynamics of a photo-generated radical pair within cryptochrome [4-6]. The hyperfine mechanism applies here despite the immobilisation of the radicals in the protein matrix, because an efficient charge-separation pathway exists that yields a well-separated radical pair in which inter-radical interactions are weak [18] compared to the dominating hyperfine interactions. In the mentioned in vitro experiments, this pair consists of a flavin adenine dinucleotide co-factor (FAD, here abbreviated as F) anion and a cation derived from the third tryptophan (W) of a highly-conserved electron transfer chain (the so-called tryptophan triad/tetrad [19]; see Figure $1 \mathrm{~A}$ ), i.e. [ $\mathrm{F}^{\bullet^{-}} \mathrm{W}^{\bullet+}$ ]. In this reaction, a spin-correlated radical pair is generated in the electronic singlet state (Figure 1B). The subsequent coherent interconversions with the corresponding triplet states arise from local hyperfine interactions and the Zeeman interaction with the external magnetic field. Eventually, the pair undergoes spin-selective reactions that discriminate the electronic spin states [4-6]. In particular, the singlet state can recombine to the diamagnetic ground state of the protein, whereas a structurally reorganized "signaling state" that initiates magnetic signal-transduction is thought to derive from all electronic spin states in a spin-insensitive reaction. This mechanism corresponds to the ideal of the RPM, which has been laid out by Kaptein \& Oosterhoff and Closs and Closs in the sense that it involves hyperfine-induced spin sorting, spin-selective recombination and "escape" (in terms of the original works) of radical pairs to form the signaling state [20-22]. Here, however, the mechanism relies on anisotropic hyperfine interactions and does not depend on the diffusive separation of the radicals for magnetosensitivity. Inter-radical interactions are present, but deemed unimportant at least in first order, in agreement with the original RPM applications [20-22].

Whether the $\left[\mathrm{F}^{\cdot-} \mathrm{W}^{\bullet+}\right]$ model applies in vivo is a matter of current debate $[1,4]$. The controversy is fueled by the realization that magnetosensitivity could actually arise in a dark-state reaction. Therefore, an alternative hypothesis focusing on the re-oxidation of the fully reduced flavin cofactor by molecular oxygen in a magnetosensitive pathway has also been considered [7, 23-26]. This would implicate a radical pair involving the swiftly relaxing superoxide anion, i.e. $\left[\mathrm{FH}^{\cdot} \mathrm{O}_{2}{ }^{--}\right]$. Magnetosensitivity is not expected in the framework of the RPM, but could still be realized in more intricate three-radical systems [27-29]. Currently, however, this alternative model still lacks the compelling evidence of a MFE realized under the strictly controlled conditions of an in vitro experiment.

In order to function as a magnetic compass sensor, the radicals require at least some degree of immobilization and alignment [30-32]. If provided, anisotropic hyperfine interactions are expected to attribute directionality to the reaction yields. So far, an orientational response has not been observed in in vitro experiments on the putatively magnetosensitive reaction pathways implicated in magnetoreception. However, a modified cryptochrome has been suggested to exhibit an anisotropic MFE at $3 \mathrm{mT}$ upon repairing cyclobutane pyrimidine dimer lesions in duplex DNA [33]. For a photolyase, an anisotropic response has even been postulated for a $40 \mu \mathrm{T}$ field. Given the reaction kinetics associated with DNA repair and the suppressive effect of large exchange coupling, it is dubious as to how this reaction could be magnetosensitive as a result of the RPM [34].

Motivated by the complexity involved with experimental studies, several theoretical approaches have aimed to further clarify the anisotropic MFEs on cryptochromes, in turn, providing predictions for reaction anisotropies in various scenarios [35-43]. Many investigations have addressed the fundamental principles and feasibility of highly simplified models for radical pair states within cryptochrome. However, considerably fewer studies have attempted to provide more realistic models, which reflect the complexity of the spin systems due to the multitude of magnetic nuclei coupled to the delocalized electron spin. Based on results from [39], $\mathrm{F}^{\cdot-}$ can be viewed as ideally suited for underpinning the directional sensitivity of cryptochrome in the presence of the geomagnetic field. This 
is facilitated by a hyperfine coupling pattern that is dominated by the two nearly axial hyperfine interactions with the N5 and N10 nitrogen atoms. Large anisotropies have been predicted when $\mathrm{F}^{\cdot-}$ is paired with a hypothetical radical, conventionally denoted $Z^{*}$; which is devoid of hyperfine interactions with magnetic nuclei, such as hydrogen and nitrogen [39]. The favorable magnetic attributes associated with this radical arise from the near-optimal magnetic properties of the flavin radical, combined with the asymmetric distribution of hyperfine interactions in the sense of a reference-probe model. Here, $\mathrm{F}^{\circ-}$ plays the role of the 'reference radical' that controls the anisotropy and $\mathrm{Z}^{\circ}$, the 'probe radical', that couples the system to the magnetic field. Although it may be tempting to identify $\mathbf{Z}^{\circ}$ as $\mathrm{O}_{2}{ }^{--}$(or dioxygen, $\mathrm{O}_{2}$ ) this presumption is encumbered by the fast spin relaxation of the latter, which impedes MFEs arising from the RPM [27]. The ascorbyl radical, Asc ${ }^{\circ}$, is thought to closely replicate the ideal properties of $Z^{\bullet}[39]$. However, in a recent study on the cryptochrome from $D$. melanogaster, no dedicated ascorbic acid binding sites could be identified that would expedite the required rapid formation of $\left[\mathrm{F}^{--} \mathrm{Asc}{ }^{\circ-}\right]$ from the photo-activated protein [44].

For the same coherent lifetime, the $\left[\mathrm{F}^{\cdot-} \mathrm{W}^{\bullet+}\right]$ radical pair is expected to be two orders of magnitude lower in sensitivity to the direction of the geomagnetic field than $\left[\mathrm{F}^{\circ-} \mathrm{Z}^{\circ}\right]$ [39]. Furthermore, the tryptophan opens up additional relaxation pathways that further attenuate the MFE by thermalizing the spin system and destroying vital coherences [37]. Despite this, it is interesting to note that level anti-crossing effects could give rise to spikey profiles of the directional recombination yield, if the spin relaxation rate was slower (by at least a factor of 10 ) than predicted, based on the dynamic characteristics of the protein [38]. This realization has attracted attention not only because the spiky reaction yields could underpin the remarkable accuracy of the avian compass, but more so because the effect is deemed truly non-trivially quantum by nature and may equip a biological system with a decisive advantage $[38,45-47]$. While the presence of many hyperfine coupling interactions without common symmetry in $\left[\mathrm{F}^{--} \mathrm{W}^{\cdot+}\right]$ attenuate the magnetosensitivity, it also makes the sensor more robust to variations in the hyperfine parameters. These arise from static inhomogeneity, which is expected to manifest within the ensemble of contributing sensory proteins in a realistic biological environment [40]. Tyrosine radicals (abbreviated as $\mathrm{Y}^{\bullet}$ ) have also been implicated within cryptochrome photo-chemistry $[19,48-50]$. However, no detailed theoretical studies of the magnetosensitivity of $\left[\mathrm{F}^{\cdot-} \mathrm{Y}^{\bullet}\right]$ have so far been presented.

For radical pairs with anisotropic hyperfine couplings in both radicals, the MFEs depend on the relative orientation of the radicals. So far, this dependency has not been investigated for realistic radical pairs (i.e. for systems with sufficient complexity to illuminate actual cryptochrome behaviors). Instead, motivated by the photocycle as informed by in vitro studies, the relevant relative orientation was presupposed to correspond to that of the FAD cofactor and the third tryptophan of the tryptophan triad/tetrad, $W_{c}$ [37-39]. The crystal structures of the cryptochrome from D. melanogaster (DmCry, PDB entry $4 G U 5, W_{C}=W 342$, see Figure $1,[51,52]$ ) and cryptochrome 1 from $A$. thaliana (AtCry, PDB entry $\left.1 \mathrm{U} 3 \mathrm{C}, \mathrm{W}_{\mathrm{C}}=\mathrm{W} 324,[53]\right)$ were used as templates. However, in view of recent reports, including: alternative electron transfer pathways in cryptochromes, structural variations induced by binding partners and small bound metabolites, and ambiguity surrounding the identity of the magnetosensitive radical pair, [54-56] a more comprehensive investigation into the dependency of the MFEs on the relative orientation of the radical sites appears desirable.

Our purpose here is to propose an approach that systematically evaluates the dependence of the singlet yields on the relative orientations of realistic cryptochrome based radical pairs. To this end, we build upon a well-established methodology, which, by neglecting inter-radical interactions, allows the formulation of reaction yields in terms of time correlation functions of the Cartesian electron spin operators of the individual radicals [57-59]. Despite the aforementioned simplifications, this approach 
can provide an adequate picture of the directional magnetosensitivity of systems involving many hyperfine-coupled nuclei. The general approach has previously been employed to model anisotropic MFEs in cryptochromes [37, 39]. Here, we extend the approach to accommodate variable relative orientations without requiring a repeated solution of the Liouville-von Neumann equation (or equivalent expressions in alternative formulations) and apply it to the $\left[\mathrm{F}^{\cdot-} \mathrm{W}^{\cdot+}\right]$ and $\left[\mathrm{F}^{-{ }^{-}} \mathrm{Y}^{\bullet}\right]$ radical pairs whilst considering all important hyperfine-coupled nuclei. We aim to provide an answer to the question as to whether the small directional effects previously found for realistic models of [ $\left.\mathrm{F}^{{ }^{--}} \mathrm{W}^{\cdot+}\right]$ are a mere consequence of an inauspicious relative orientation of the radicals selected for study, or reflect a more general limitation of the system. Furthermore, we hope that our systematic study of relative orientations might inspire an optimized cryptochrome design for magnetosensitivity with specific magneto-optogenetic approaches in mind, e.g. by dedicated mutations. In fact, Cry has previously been used as an optogenetic tool [60] and can render the activity of a system that do not naturally contain Cry magnetically-sensitive [61]. Additionally this research could guide endeavors to experimentally observe anisotropic MFEs in cryptochromes in vitro or in chemically engineered model compounds such as that reported in $[62,63]$. Finally, our study will provide indirect insight into the current debate surrounding the identity of the crucial radical pair, by providing a more complete picture of the magnetosensitivity of $\left[\mathrm{F}^{\cdot-} \mathrm{W}^{\cdot+}\right]$ and $\left[\mathrm{F}^{\cdot-} \mathrm{Y}^{*}\right]$. We shall furthermore comment on the significance of entanglement to radical pair magnetoreception in these model radicals.

\section{Theory}

The spin dynamics of the radical pair shall be described in terms of the spin density operator $\hat{\rho}(t)$. For rigidly immobilized radical pairs undergoing first-order singlet and triplet recombination reactions at the rates $k_{\mathrm{S}}$ and $k_{\mathrm{T}}$, respectively, the corresponding equation of motion reads:

$$
\frac{\mathrm{d} \hat{\rho}(t)}{\mathrm{d} t}=-i[\hat{H}, \hat{\rho}(t)]-\frac{k_{S}}{2}\left[\hat{P}_{s}, \hat{\rho}(t)\right]-\frac{k_{T}}{2}\left[\hat{P}_{T}, \hat{\rho}(t)\right] .
$$

Here, $\hat{P}_{S}$ and $\hat{P}_{T}$ are the singlet and triplet projection operators, respectively. Spin-selective reactions have been accounted for by the Haberkorn approach [64] and spin relaxation has been neglected [3537]. Our treatment, thus, applies to radical pairs that are short-lived compared to their spin relaxation times. Assuming that the coherent evolution is dominated by the hyperfine interactions between the electron and nuclear spins within each radical, in combination with the Zeeman interactions between the electron spins and an applied magnetic field, the Hamiltonian is of the form:

$$
\hat{H}=\hat{H}_{\mathrm{A}}+\hat{H}_{\mathrm{B}} \text { with } \hat{H}_{\mathrm{i}}=\sum_{j} \hat{\mathbf{S}}_{i} \cdot \mathbf{A}_{i, j} \cdot \hat{\mathbf{i}}_{i, j}+\vec{\omega}_{0} \cdot \hat{\mathbf{S}}_{i} \cdot
$$

The Zeeman precession frequency is given by $\vec{\omega}_{0}=-\gamma_{i} \vec{B}_{0}$, where $\gamma_{i}$ denotes the gyromagnetic ratio of the electron in radical $i \in\{A, B\}$ and $\vec{B}_{0}$ the applied magnetic field. $\mathbf{A}_{i, j}$ is the hyperfine coupling tensor between the $j$ th nuclear spin and the ith electron spin; $\hat{\mathbf{i}}_{i, j}$ and $\hat{\mathbf{S}}_{i}$ are the corresponding vector operators of nuclear and electron spin angular momentum.

We assume that a radical pair is created in the electronic singlet state, i.e.

$$
\hat{\rho}(0)=\hat{P}_{S} / \operatorname{Tr}\left[\hat{P}_{S}\right]=\frac{1}{z_{A} z_{B}} \hat{P}_{S}
$$

where $z_{i}=\prod_{j}\left(2 l_{i, j}+1\right)$ is the dimension of the nuclear Hilbert space in radical $i$. The concentrationweighted probability of finding the radical pair in the singlet state at time $t$ is calculated from 


$$
n_{\mathrm{s}}(t)=\operatorname{Tr}\left[\hat{P}_{s} \hat{\rho}(t)\right]
$$

The total amount of product generated via the singlet channel once all radical pairs have recombined is

$$
\Phi_{\mathrm{s}}=k_{\mathrm{s}} \int_{0}^{\infty} n_{\mathrm{s}}(t) \mathrm{d} t
$$

Solving eq. (1) is a formidable task for long-lived radical pairs with a large number of hyperfinecoupled nuclei, which is to be repeated for a multitude of magnetic field directions and, given our objective, for numerous relative orientations of the radicals. Here, we shall employ an approach which rests upon two simplifying assumptions: Firstly, we assume that the effect of inter-radical interactions can be neglected (such as exchange and electron-electron dipolar coupling [65]), as already assumed in stating eq. (2). Secondly, we postulate that the singlet and triplet reaction rates are equal. Neither of these assumptions strictly applies, yet, they are widely applied in this context to render the task of solving eq. (1) for realistic radical pairs manageable without resorting to semi-classical approximations. In a recent paper, Hore provides a detailed assessment of these approximations in the context of cryptochrome [66]. For the parameter ranges typically expected, these omissions are considered tolerable. We will further comment on these assumptions in the discussion.

In the limit that $k_{\mathrm{S}}=k_{\mathrm{T}}=k$ and for commuting $\hat{H}_{\mathrm{A}}$ and $\hat{H}_{\mathrm{B}}$, the singlet probability can be expressed in terms of the correlation functions associated with the Cartesian spin operators of the individual radicals,

$$
T_{\alpha, \beta}^{(i)}(t)=\frac{1}{z_{i}} \operatorname{Tr}\left[\hat{S}_{i, \alpha} \hat{S}_{i, \beta}(t)\right]=\frac{1}{z_{i}} \operatorname{Tr}\left[\hat{S}_{i, \alpha} e^{+i \hat{H}_{i},} \hat{S}_{i, \beta} e^{-i \hat{H}_{i} t}\right],
$$

which, for $\alpha, \beta \in\{x, y, z\}$, assemble the spin correlation tensors $\mathbf{T}^{(i)}(t)$ [57]. Here, $S_{i, \alpha}(t)$ is the spin operator in the Heisenberg picture. Specifically, using $\hat{P}_{S}=\frac{1}{4}-\hat{\mathbf{S}}_{A} \cdot \hat{\mathbf{S}}_{B}$ and the above-stated assumptions in eq. (1), one finds that

$$
n_{\mathrm{s}}(t)=p_{s}(t) \exp (-k t)
$$

where the singlet probability $p_{s}(t)$ is given by

$$
p_{S}(t)=\frac{1}{z_{A} z_{B}} \operatorname{Tr}\left[\hat{P}_{S} \hat{P}_{S}(t ; k=0)\right]=\frac{1}{4}+\sum_{\alpha, \beta} T_{\alpha, \beta}^{(A)}(t) T_{\alpha, \beta}^{B}(t)
$$

All time dependent quantities here relate to the $k=0$ scenario; the reaction enters in the form of an exponential scaling of eq. (7). Eq. (8) allows the calculation of recombination yields for reasonably complex radical pairs, because the problem is reduced to the independent evaluation of the spin correlation tensors for the individual radicals, i.e. two independent calculations. Yet, it does not provide an efficient pathway leading to the evaluation of relative radical orientations, because, as it stands, the spin correlation tensor of the reoriented radical ought to be re-calculated for every orientation. In the Appendix, we show that this, however, is not required, as

$$
\mathbf{T}^{(i)}\left(t ; \vec{B}_{0},\left\{\mathbf{R} \mathbf{A}_{i, j} \mathbf{R}^{-1}\right\}\right)=\mathbf{R} \mathbf{T}^{(i)}\left(t ; \mathbf{R}^{-1} \vec{B}_{0},\left\{\mathbf{A}_{i, j}\right\}\right) \mathbf{R}^{-1} .
$$


Here, $\mathbf{T}^{(i)}\left(t ; \vec{B}_{0}, \mathbf{A}_{j}\right)$ denotes the spin correlation tensor for a given magnetic field, $\vec{B}_{0}$, and set of hyperfine tensor parameters, $\mathbf{A}_{j} . \mathbf{R}$ is a rotation matrix and $\mathbf{T}^{(i)}\left(t ; \vec{B}_{0},\left\{\mathbf{R} \mathbf{A}_{j} \mathbf{R}^{-1}\right\}\right)$ the spin correlation tensor of the radical with rotated orientation. In words, eq. (9) suggests that instead of rotating the hyperfine tensors, the spin correlation tensors could be reoriented if the magnetic field is also simultaneously counter-rotated. In combination with the evaluation of spin correlation tensors for a grid of magnetic field orientations, this provides an efficient approach to calculate $p_{s}(t)$ for cases that the evaluation of the time-dependence of $\mathbf{T}^{(i)}$ is the limiting factor, i.e. for radicals with large numbers of hyperfine terms and/or if their lifetimes are considered long.

To assess entanglement of the electron spins, we shall reconstruct the electronic density operator, $\hat{\rho}_{e l}(t)$, from the spin correlation tensors. To this end, we express $\hat{\rho}(t)$ in a complete, orthogonal operator basis $\left\{\hat{\Omega}_{i}\right\}$ comprising the identity $\hat{1}$, the Cartesian spin operators $\left\{\hat{S}_{i, \alpha}\right\}$, and their bi-linear combinations for radicals $A$ and $B\left\{\hat{S}_{A, \alpha} \hat{S}_{B, \beta}\right\}$ :

$$
\hat{\rho}_{e l}(t)=\operatorname{Tr}_{n}[\hat{\rho}(t)]=\sum_{i=1}^{16} c_{i}(t) \hat{\Omega}_{i}
$$

Here, $T r_{n}$ denotes the trace over nuclear degrees of freedom and the $c_{i}(t) s$ are time-dependent expansion coefficients. For the singlet initial configuration, the only non-zero expansion coefficients are associated with $\hat{1}$ and the bilinear terms, $\left\{\hat{S}_{A, \alpha} \hat{S}_{B, \beta}\right\}$. For the latter we find:

$$
\frac{1}{4} c_{i}(t)=\left\langle\hat{S}_{A, \alpha} \hat{S}_{B, \beta}\right\rangle(t)=-\sum_{\gamma \in\{x, y, z\}} T_{\gamma \alpha}^{A}(t) T_{\gamma \beta}^{B}(t) \equiv-Q_{\alpha \beta}(t) \quad \text { for } \quad \hat{\Omega}_{i} \in\left\{\hat{S}_{A, \alpha} \hat{S}_{B, \beta}\right\}
$$

Here we have assumed $k=0$; the decay can always be reinstated by multiplying with $\exp (-k t)$. The expansion coefficient associated with $\hat{1}$ is $1 / 4$. We will use the expansion eq. (11) to assess the entanglement of the electron spins from the eigenvalues of the partial transpose of the first radical, $\hat{\rho}_{e l}^{T_{A}}(t)[67,68]$. The partial transpose can be calculated from eq. (10) by using the fact that $\hat{S}_{y}$ is antisymmetric to transposition, whereas the real operators are symmetric. Thus, by exchanging the sign of all expansion coefficients of $\hat{S}_{A, y}$ containing operators, we obtain:

$$
\hat{\rho}_{e l}^{T_{A}}(t)=\left(\begin{array}{cccc}
\frac{1}{4}-Q_{z z} & -Q_{z x} & -Q_{x z} & -Q_{x x}-Q_{p y} \\
\vdots & \frac{1}{4}+Q_{z z} & Q_{p y}-Q_{z x} & Q_{x z} \\
& \vdots & \frac{1}{4}+Q_{z z} & Q_{z x} \\
\ldots & & \cdots & \frac{1}{4}-Q_{z z}
\end{array}\right)+i\left(\begin{array}{cccc}
0 & Q_{z y} & -Q_{y z} & Q_{x y}-Q_{y z} \\
\vdots & 0 & -Q_{x y}-Q_{p x} & Q_{y z} \\
& \vdots & 0 & -Q_{z y} \\
\ldots & & \ldots & 0
\end{array}\right)
$$

The matrix elements that are not given explicitly follow from the Hermiticity of $\hat{\rho}_{e l}^{T_{A}}(t)$. Based on the Peres-Horodecki criterion, the partial transpose is positive if the density matrix is separable $[67,68]$. For $2 \times 2$-systems like the one studied here, entangled states are conversely indicated by negative eigenvalues of $\hat{\rho}_{e l}^{T_{A}}(t)$. This observation motivates the definition of negativity as a measure of quantum entanglement [69]:

$$
\mathcal{N}\left(\hat{\rho}_{e l}\right)=\sum_{i} \frac{\left|\lambda_{i}\right|-\lambda_{i}}{2}
$$


where the $\lambda_{i} s$ are the eigenvalues of $\hat{\rho}_{e l}^{T_{A}}(t) . \mathcal{N}\left(\hat{\rho}_{e l}\right)$ equals the absolute sum of the negative eigenvalues. It is an entanglement monotone. The evaluation of $\hat{\rho}_{e l}^{T_{A}}(t)$ from spin correlation tensors will allow us to evaluate negativities of large spin systems. It is interesting to note that mixtures of singlet and unpolarized triplet states are separable unless they contain more than a $3 / 4$-share of the singlet state [70]. Furthermore, the negativity of a reaction stemming from the unpolarized triplet configuration can be calculated from that given for the singlet configuration above as $\hat{\rho}_{e l, T}^{T_{A}}=\frac{1}{2} \hat{l}-\hat{\rho}_{e l, S}^{T_{A}}$ . Here, the additional subscripts label the initial spin state. Consequently, for the triplet-born radical pair the negativity is $\mathcal{N}_{T}\left(\hat{\rho}_{e l}\right)=\frac{1}{2} \sum_{i}\left|\frac{1}{2}-\lambda_{s, i}\right|-\left(\frac{1}{2}-\lambda_{s, i}\right)$.

To quantify ST-coherences [71], we use the $I_{1}$-norm measure

$$
C\left(\hat{\rho}_{e l}\right)=\sum_{\substack{i, j=\left\{s, T_{0}, T_{+}, T_{-}\right\} \\ i \neq j}}\left|\left\langle i\left|\hat{\rho}_{e l}\right| j\right\rangle\right|,
$$

with the electronic density matrix $\hat{\rho}_{e l}(t)$ calculated from the spin correlation tensors according to eq. (10).

\section{Methods}

We have numerically evaluated the spin correlation tensors for the radical cation of tryptophan (9 nuclear spins), the oxyl radical of tyrosine (7 nuclear spins) and the flavin anion radical (12 nuclear spins) on an equidistant time grid, $t_{n}=n \Delta t$. In Figure 2 , the chemical structures and hyperfine interactions of the studied radicals are shown in their respective molecular axes systems (defined in Figure S4 in the SI); hyperfine parameters are summarized in the Supporting Information (Tables S1 S3). The geomagnetic field intensity was taken to be $50 \mu \mathrm{T}$ with its orientation varied on a geodesic sphere resulting from the tessellation of an icosahedron (10242 orientations forming 20480 triangular faces). As the MFE is invariant to inversion, practically only half the number of orientations corresponding to a hemisphere require evaluation. A rate constant of $k=10^{6} \mathrm{~s}^{-1}$ was assumed in agreement with the order of magnitude of the rate constants observed in in vitro experiments on the isolated proteins $[14,15]$. The maximal time was $25 k^{-1}$ and $2^{15}$ time steps were used ( $\left.\Delta t \approx 0.76 \mathrm{~ns}\right)$. In order to evaluate the spin correlation functions, we re-express eq. (6) in the eigenbasis of the Hamiltonian as

$$
T_{\alpha, \beta}^{(i)}(n \Delta t)=\sum_{\substack{a, b=1 \\ A_{a b} \neq 0}}^{N} A_{a b} B_{a b}^{n},
$$

where $A_{a b}=\left\langle a\left|\hat{S}_{i, \alpha}\right| b\right\rangle\left\langle b\left|\hat{S}_{i, \beta}\right| a\right\rangle, \quad B_{a b}=\exp \left(i\left(\omega_{b}-\omega_{a}\right) \Delta t\right)$ with $\omega_{a}$ denoting the angular frequency associated with the $a$ th eigenstate $|a\rangle$, and $N$ is the dimension of the Hilbert space. Eq. (15) requires an element-wise vector multiplication and a sum reduction in every time marching step. We use CUDA to efficiently implement this process on GPUs. On our hardware (NVidia Tesla K80 GPU and Intel Xeon CPU E5-2640 v3 @ 2.60GHz) this affords a 6.5-time speedup over our CPU implementation for the flavin anion radical. 
We study the relative orientional dependence of the two radicals by rotating the flavin radical anion with respect to the other. Sparing the intensive time-propagation step, the spin correlation tensor of the rotated radical is evaluated from eq. (9), i.e. by transforming $\mathbf{T}^{(B)}\left(t ; \mathbf{R}^{-1} \vec{B}_{0},\left\{\mathbf{A}_{B, j}\right\}\right)$. As this quantity refers to the counter-rotated magnetic field $\mathbf{R}^{-1} \vec{B}_{0}$, it usually has to be evaluated by interpolation from the orientations available on the constructed spherical grid. For the calculation of $\Phi_{s}\left(\vec{B}_{0}\right)$, this process can be considerably sped up by reformulating eq. (5) as

$$
\begin{aligned}
\Phi_{S}\left(\vec{B}_{0}\right) & =\frac{1}{4}+k \int_{0}^{\infty} \exp (-k t)\left(\mathbf{T}^{(A)}(t): \mathbf{R} \mathbf{T}^{(B)}\left(t ; \mathbf{R}^{T} \vec{B}_{0},\left\{\mathbf{A}_{B, j}\right\}\right) \mathbf{R}^{T}\right) \mathrm{d} t \\
& =\frac{1}{4}+k \int_{0}^{\infty} \exp (-k t) \operatorname{vec}\left(\mathbf{T}^{(A)}(t)\right)^{T}(\mathbf{R} \otimes \mathbf{R}) \operatorname{vec}\left(\mathbf{T}^{(B)}\left(t ; \mathbf{R}^{T} \vec{B}_{0},\left\{\mathbf{A}_{B, j}\right\}\right)\right) \mathrm{d} t \\
& =\frac{1}{4}+\sum_{l . m=1}^{9}(\mathbf{R} \otimes \mathbf{R})_{l, m} \mathbf{X}_{l . m}\left(\vec{B}_{0}, \mathbf{R}^{T} \vec{B}_{0}\right)
\end{aligned}
$$

where $\mathbf{X}_{l . m}\left(\vec{B}_{0}, \mathbf{R}^{\top} \vec{B}_{0}\right)$ denotes a $9 \times 9-$ matrix defined by

$$
\mathbf{X}_{l . m}\left(\vec{B}_{0}, \mathbf{R}^{T} \vec{B}_{0}\right)=k \int_{0}^{\infty} \exp (-k t) \operatorname{vec}\left(\mathbf{T}^{(A)}\left(t ; \mathbf{R}^{T} \vec{B}_{0}\right)\right), \operatorname{vec}\left(\mathbf{T}^{(B)}\left(t ; \mathbf{R}^{T} \vec{B}_{0}\right)\right)_{m} \mathrm{~d} t
$$

Here, vec denotes the vectorization of a matrix. This approach is significantly more efficient for calculating $\Phi_{s}\left(\vec{B}_{0}\right)$, because it interchanges the time integration and the interpolation. It necessitates 81 interpolations of the components of $\mathbf{X}_{l, m}\left(\vec{B}_{0}, \mathbf{R}^{T} \vec{B}_{0}\right)$ instead of a separate interpolation for every $t$ value, i.e. here, $2^{15}+1$ interpolations.

We have systematically scanned all three Euler angles that define the relative orientation of the radicals. For this extensive search, we have used a linear interpolation on the triangular faces of the spherical grid expressed in barycentric coordinates. The intersection of $\mathbf{R}^{\top} \vec{B}_{0}$ with the mesh triangles was calculated using the Möller-Trumbore algorithm [72]. For more detailed calculations on individual orientations we have employed radial basis functions on the sub-grids of 11 or 12 points that cover the triangle intersecting $\mathbf{R}^{T} \vec{B}_{0}$ and one layer of adjacent triangles. Gaussian basis functions and a distance function based on the great circle distance were used. The MFEs calculated from these two approaches differed only in the fifth significant digit.

As we pre-calculate the spin correlations for a hemi-spherical grid of orientations, the calculation of MFEs due to reoriented radicals occasionally requires $\mathbf{T}^{(B)}\left(t ; \mathbf{R}^{T} \vec{B}_{0},\left\{\mathbf{A}_{B, j}\right\}\right)$ for orientations $\mathbf{R}^{T} \vec{B}_{0}$ outside of the covered hemisphere. These missing tensors can be reconstructed from the tensors for the inverted field, $-\mathbf{R}^{T} \vec{B}_{0}$ as follows:

$$
\mathbf{T}^{(i)}\left(t ;-\vec{B}_{0},\left\{\mathbf{A}_{i, j}\right\}\right)=\mathbf{T}^{(i)}\left(-t ; \vec{B}_{0},\left\{\mathbf{A}_{i, j}\right\}\right)=\left(\mathbf{T}^{(i)}\left(t ; \vec{B}_{0},\left\{\mathbf{A}_{i, j}\right\}\right)\right)^{T} .
$$

Here, the first identity results from the fact that the Hamiltonian, eq. (2), is symmetric under timereversal and simultaneous inversion of the magnetic field. The second equality results from the fact that for the spin correlation tensors, time-reversal is tantamount to transposition, which is evident from the definition in eq. (6). Note that, unlike the singlet yield, the spin correlation tensors are not symmetric under field inversion. 


\section{Results}

We have used the methodology introduced above to evaluate the singlet yield for a range of radical pairs involving the flavin anion radical $\left(\mathrm{F}^{\bullet^{-}}\right)$, the tryptophan radical cation $\left(\mathrm{W}^{\bullet+}\right)$ and the neutral tyrosine radical $\left(\mathrm{Y}^{\bullet}\right)$. Additionally, inspired by previous works [39], we considered scenarios in which these radicals are combined with a hypothetical radical, conventionally denoted $Z^{*}$, that is devoid of hyperfine-coupled nuclear spins. The largest and most significant hyperfine coupling constants have been included (see Figure 2): 12 coupled nuclei for $\mathrm{F}^{\bullet-}, 9$ for $\mathrm{W}^{*+}$, and 7 for $\mathrm{Y}^{\bullet}$. The hyperfine tensors, which are listed in the SI (Tables S1 to S3), were calculated by density functional theory using the UB3LYP/6-31+G(d,p)//UB3LYP/EPR-III protocol in Gaussian 16 [73]. The (isotropic) hyperfine interactions of the methylene groups adjacent to the aromatic rings of the radicals ( $\beta$-protons) depend on the dihedral angle that the $\mathrm{C}-\mathrm{H}$-bonds make with the normal to the aromatic plane (as described in good approximation by the Heller-McConnell equation) [74]. For $\mathrm{F}^{{ }^{-}}$this dihedral was fixed to yield the conformer as found for the FAD cofactor in the crystal structure of the Drosophila melanogaster cryptochrome (DmCry, PDB entry 4GU5; dihedral angle CD1-CG-CB-CA: $\left.-74.9^{\circ},[51,52]\right)$. For $\mathrm{W}^{\circ+}$ and $\mathrm{Y}^{\bullet}$ representative rotamers were chosen; the arguments underpinning this selection process are detailed in the $\mathrm{SI}$. For $\mathrm{W}^{\bullet+}$, the chosen conformer resembled $\mathrm{W}_{\mathrm{C}}$ in $\mathrm{DmCry}$ (with CA-CB-CG-CD1 dihedral angle of $103.7^{\circ}$ ).

In order to quantify the effectiveness of a radical pair to act as a magnetic compass we introduce two metrics: the absolute and relative anisotropy, $\Delta_{s}$ and $\Gamma_{s}$, respectively. They are defined by

$$
\Delta_{s}=\max _{\theta, \phi} \Phi_{s}\left(\vec{B}_{0}(\theta, \phi)\right)-\min _{\theta, \phi} \Phi_{s}\left(\vec{B}_{0}(\theta, \phi)\right) \quad \text { and } \quad \Gamma_{s}=\frac{\Delta_{s}}{\left\langle\Phi_{s}\right\rangle}
$$

where $\theta$ and $\phi$ evaluate all directions of the magnetic field vector in the molecular frame of the radical pair and $\left\langle\Phi_{s}\right\rangle$ denotes the mean yield.

\section{Truncated radical pairs at the geomagnetic field $(50 \mu T)$}

The radical pairs involving the hypothetical $\mathrm{Z}^{\bullet}$ in combination with $\mathrm{F}^{\bullet-}$ and $\mathrm{W}^{\bullet+}$ have already been addressed in an earlier study [39]. For comparison, we shall briefly summarize our findings in relation to these previous results. Our models differ from those of [39] as they consider more hyperfinecoupled nuclear spins, both in $\mathrm{F}^{\bullet-}$ and $\mathrm{W}^{*+}$. Additionally, they include a different choice of sidechain conformations, which predominantly impact the $\beta$-hyperfine coupling constants. For $\left[\mathrm{F}^{\circ-} Z^{\circ}\right]$, we find $\Delta_{S}=0.147\left(\Gamma_{\mathrm{S}}=47.8 \%\right)$, which is in close agreement with the previous results $\left(\Delta_{\mathrm{S}}=0.146\right)$. This observation once again reinforces the interpretation that in $\mathrm{F}^{\circ-}$, the spin dynamics are governed by the two dominant, nearly axial hyperfine interactions at $\mathrm{N} 5$ and $\mathrm{N} 10$ and are widely insensitive to the remaining hyperfine terms. On the other hand, for $\left[Z^{\bullet} \mathrm{W}^{\bullet+}\right]$, we obtain an anisotropy of $\Delta_{s}=0.030\left(\Gamma_{\mathrm{s}}\right.$ $=11.2 \%)$, which is markedly smaller by more than a factor of two than that reported in [39] $\left(\Delta_{\mathrm{S}}=\right.$ 0.062 ). The reduced compass sensitivity of the $\mathrm{W}^{\bullet+}$-containing radical pairs has been attributed to the many hyperfine interactions within $\mathbf{W}^{*+}$ that are of comparable size, but lack common symmetries. Our results suggest that the additional hyperfine interactions considered here ( $\mathrm{H} 5$ and $\mathrm{H} \beta 2$ have been included in addition) perpetuate this trend. It is noteworthy that the shape of the anisotropic responses remain largely unaltered. Here, the anisotropies of both, $\left[\mathrm{F}^{\bullet-} Z^{*}\right]$ and $\left[Z^{\bullet} \mathrm{W}^{\bullet+}\right]$, are found to be of the functional form $\Phi_{s}\left(\vec{B}_{0}(\theta, \phi)\right) \approx c_{0,0} Y_{0,0}(\theta, \phi)+c_{2,0} Y_{2,0}(\theta, \phi)$, with the coefficients of the nextlargest spherical harmonics, $Y_{2, \pm 1}(\theta, \phi)$, contributing less than one thousandth of the $Y_{2,0}(\theta, \phi)$ - 
contribution (as assessed by the absolute values squared of the expansion coefficients $c_{k, l}$; see Figure $\mathrm{S} 3$ in the SI). Note, however, that this simple dependence on the orientation of the radical pair does not apply for all magnetic field intensities, e.g. for $\left[\mathrm{Z}^{\bullet} \mathrm{W}^{*+}\right]$ at $B_{0}=1 \mathrm{mT}$, a markedly more complex pattern ensues.

We have also considered the $\left[\mathrm{Z}^{\bullet} \mathrm{Y}^{\bullet}\right]$ radical pair, where $\mathrm{Y}^{\bullet}$ refers to the tyrosine phenolic radical and $\mathrm{Z}^{\bullet}$ the "free electron" as above. The magnetosensitivity of $Y^{\bullet}$-based radical pairs has not previously been considered in the context of magnetoreception. As illustrated in Figure 2, the hyperfine structure of $\mathrm{Y}^{\bullet}$ differs from that of $\mathrm{F}^{\bullet-}$ or $\mathrm{W}^{\bullet+}$. It is characterized by a greater simplicity, fewer coupled nuclear spins and the lack of aromatic nitrogen hyperfine couplings with characteristic $p$-orbital-like shape, as observed for $\mathrm{N} 5$ and $\mathrm{N} 10$ in $\mathrm{F}^{\bullet-}$ and $\mathrm{N} 1$ in $\mathrm{W}^{*+}$. The proton hyperfine interactions are the dominant carriers of hyperfine anisotropy. Interestingly, on its own, the $\mathrm{Y}^{\bullet}$ hyperfine structure does not evoke large anisotropies of the MFE. We find a small $\Delta_{s}=0.014\left(\Gamma_{s}=5.3 \%\right)$, which is smaller than that of $\left[\mathrm{Z}^{*} \mathrm{~W}^{*+}\right]$ by a factor of approximately 2 . The shape of the anisotropy, which is shown in Figure $\mathrm{S} 3$ in the $\mathrm{SI}$, is also different from the patterns displayed above. A cross-shaped figure results for $\left[\mathrm{Z}^{\bullet} \mathrm{Y}^{\bullet}\right]$ from larger $Y_{2, \pm 2}(\theta, \phi)$ contributions in the spherical harmonic expansion besides the dominant $Y_{2,0}(\theta, \phi)$ $\left(c_{0,0}{ }^{2} /\left|c_{2, \pm 2}\right|^{2} \sim 8\right)$.

\section{Flavin-tryptophan radical pairs with variable relative orientation in the geomagnetic field}

Following the approach as outlined above, we have evaluated the singlet anisotropies of $\left[\mathrm{F}^{\cdot-} \mathrm{W}^{\bullet+}\right]$ as a function of the relative orientations of the two radicals. To this end, the flavin radical anion was rotated relative to the molecular frame of the radical pair, which was taken to coincide with the molecular axis system of tryptophan as indicated in Figure $\mathrm{S} 1$ or 2 . The rotation was parametrized by the three Euler angles $\alpha, \beta, \gamma$ in the zxz-convention. As multiple, ambiguous conventions are in use, we make the definition of the rotation matrix available in terms of its constituent Euler angles in Appendix 2 (cf. Figure S2). Here, we have systematically explored the relative orientations by evaluating the singlet anisotropies on a regular grid covering 120 points each in $\alpha$ and $\gamma \in[0,2 \pi)$, and 60 points in $\beta \in[0, \pi)$, i.e. the angular resolution was $3^{\circ}$. The evaluation of the resulting 864,000 relative orientations is made possible for large spin systems by the algorithm described above, in particular eqs. (9) and (16). We have opted to rotate $\mathrm{F}^{\circ-}$, because the form of the hyperfine tensors arising from the two dominant nitrogens ( $\mathrm{N} 5$ and $\mathrm{N} 10$ ), promised a minor dependency on the angles $\alpha$ and $\gamma$, for which the rotation occurs about the principal axis of these tensors.

Heat maps of the relative singlet yield anisotropy, $\Gamma_{\mathrm{s}}$, at $50 \mu \mathrm{T}$ as a function of relative orientation can be seen in Figure 3. The highest relative anisotropy was $\Gamma_{\mathrm{S}}=0.27 \%$, attained for the Euler angles $\alpha=86^{\circ}, \beta=5^{\circ}$ and $\gamma=107^{\circ}$. The corresponding $\Delta_{s}$ amounts to $7.4 \times 10^{-4}$. The heat maps reveal that variation in anisotropy are predominantly moderated by the $\beta$ orientational degree of freedom, which characterizes the tilt of the aromatic ring planes in $\mathrm{F}^{\circ-}$ and $\mathrm{W}^{\bullet+}$. Large anisotropies are generally associated with close to parallel or antiparallel orientations of the perpendiculars to these ring planes. The $\alpha$ and $\gamma$ degree of freedom induce only minor modulations of this dominating motif. The maximal standard deviation of the $\Gamma_{\mathrm{s}}$ for a given $\beta$, but variable $\alpha$ and $\gamma$, is $1.2 \times 10^{-4}$, which corresponds to only $8.0 \%$ of the mean $\Gamma_{s}$. In particular, the dependence on $\gamma$ is virtually absent. The minimal relative anisotropy amounts to $\Gamma_{S}=0.091 \%$ and is associated with the orientation $\alpha=158^{\circ}, \beta=107^{\circ}$ and $\gamma=$ $156^{\circ}$. An analysis based on $\Delta_{\mathrm{s}}$, instead of $\Gamma_{\mathrm{s}}$, portrays a similar picture (heat maps of $\Delta_{\mathrm{s}}$ are provided in Figure S4). The maximal $\Delta_{S}=7.4 \times 10^{-4}$ is found at $\alpha=84^{\circ}, \beta=4^{\circ}$ and $\gamma=109^{\circ}$, i.e. for a relative orientation close to the optimal orientation for $\Gamma_{\mathrm{s}}$. 
We have also used the approach to explicitly evaluate the singlet anisotropy for the relative orientations as found for the tryptophan triad/tetrad in published crystal structures. $W_{c}$ is of particular interest as it forms part of the magnetosensitive radical pair identified through in vitro experiments on the purified cryptochromes of $A$. thaliana and $D$. melanogaster. For the relative orientation of the radicals, as found for the FAD cofactor and Trp-342 $\left(\mathrm{W}_{c}\right)$ in the crystal structure of DmCry, we find $\Delta_{s}$ $=4.9 \times 10^{-4}$ and $\Gamma_{S}=0.18 \%$. These values, which have here been evaluated for the radical pair with 21 hyperfine interactions included, are smaller than those provided for 14 hyperfine interactions in an earlier study $\left(\Delta_{s}=0.0014\right)$ [39]. This once again corroborates the observation that for $\left[\mathrm{F}^{\cdot-} \mathrm{W}^{*+}\right]$ the compass sensitivity deteriorates with the addition of more nuclei, in particular in $\mathrm{W}^{\bullet+}$. It is noteworthy that the shape of the anisotropic part of the singlet yield as a function of orientation found here (see Figure S5) closely resembles that of $\left[\mathrm{F}^{\cdot-} \mathrm{Z}^{\circ}\right]$ and, thus, differs from that in [39]. This is not unexpected, as the singlet yield anisotropy of the intact radical pair, $\left[\mathrm{F}^{\bullet-} \mathrm{W}^{\bullet+}\right]$, appears to be an approximate composite of the patterns for $\left[\mathrm{F}^{\bullet-} \mathrm{Z}^{*}\right]$ and $\left[\mathrm{Z}^{*} \mathrm{~W}^{*+}\right]$. As the latter here is additionally attenuated by the inclusion of additional hyperfine interactions, an anisotropy pattern more closely resembling $\left[\mathrm{F}^{\cdot-} \mathrm{Z}^{\circ}\right]$ is obtained, i.e. the effect of $\mathrm{N} 1$ from $\mathrm{W}^{*+}$ is no longer obvious.

Comparing $\left[\mathrm{F}^{\cdot-} \mathrm{W}_{\mathrm{c}}{ }^{\cdot+}\right]$ with (hypothetical) radical pairs involving any other of the 16 tryptophan residues in $D m C r y$, we find relative anisotropies ranging from $0.1 \%$ to $0.22 \%$ (see Table S4). While a functional relevance of the majority of these alternative radical pairs is obviously questionable, this comparison stresses that the radical pair with $\mathrm{W}_{\mathrm{c}}$ is, in fact, among the most sensitive radical pairs. It is only outclassed by the radical pairs with W314 and W353, for which $\Gamma_{S}=0.22 \%$.

We have also compared the tryptophans of the triad/tetrad for the established crystal structures of cryptochromes with bound flavin and a homology model of cryptochrome 4 from the European Robin $[75,76]$. The magnetic field effect anisotropies have been summarized in Table 1 . Subsequent comparison of the four/three tryptophan orientations yields the following observations: For the systems with a tryptophan tetrad, the anisotropy is always maximal for $W_{c}$. The largest anisotropy is seen for $W_{c}$ in DmCry. For the animal-like Cryptochrome from Chlamydomonas reinhardtii (PDB ID: 5ZMO) [77] and the model of the avian cryptochrome 4 [75, 76], a smaller anisotropy of $0.16 \%$ and $0.15 \%$ is found, respectively. All other relative orientations yield anisotropies of the order of $0.1 \%$. Likewise, for the plant cryptochrome 1 from $A$. thaliana, a relative anisotropy of approximately $0.1 \%$ is predicted for all positions, including the terminal $W_{c}$, both with and without bound ATP analogue.

Flavin-tyrosine radical pairs with variable relative orientation in the geomagnetic field We have studied the orientational dependence of the anisotropy for radical pairs comprising the flavin anion radical in combination with the neutral, i.e. deprotonated, tyrosine radical. The approach described above was followed, whereby the dominant 7 hyperfine interactions of $Y^{\bullet}$ were taken into account. Heat maps of the orientational dependence are shown in Figure 4. Relative anisotropies range from $\Gamma_{s}=0.18 \%$ to $0.96 \%$. Different from $\left[\mathrm{F}^{\cdot-} \mathrm{W}^{*+}\right]$, here, the largest anisotropies are associated with $\beta \sim 90^{\circ}$, i.e. the perpendicular orientation of the aromatic ring planes. The global maximum of $\Gamma_{\mathrm{s}}$ is observed for $\alpha=179^{\circ}, \beta=88^{\circ}$ and $\gamma=174^{\circ}$. The corresponding anisotropy pattern is shown in Figure $\mathrm{S6}$; it reveals a more vivid, although not more spiky, pattern than those found for $\left[\mathrm{F}^{{ }^{-}} \mathrm{W}^{\circ+}\right]$ above. As for the tryptophan-based system, the anisotropy is hardly dependent on $\gamma$ and varies little with $\alpha$. Furthermore, just as above, the absolute and the relative anisotropies show qualitatively the same orientational dependence. $\Delta_{s}$ ranges from $4.6 \times 10^{-4}$ to $2.5 \times 10^{-3}$. In general, the larger anisotropies suggests that $\left[\mathrm{F}^{{ }^{-}} \mathrm{Y}^{\bullet}\right]$ might be better versed to underpin a compass sense than $\left[\mathrm{F}^{\cdot-} \mathrm{W}^{\bullet+}\right]$. In particular, for $\left[\mathrm{F}^{\cdot-} \mathrm{Y}^{*}\right]$ the maximal relative anisotropy is larger by a factor of 3.6 than for $\left[\mathrm{F}^{{ }^{-}-} \mathrm{W}^{*+}\right]$ and even the least sensitive relative orientations are predicted to show a compass sensitivity comparable to that of the rather performant $\left[\mathrm{F}^{\cdot-} \mathrm{W}_{\mathrm{c}}^{\cdot+}\right]$ in DmCry. 


\section{Radical pairs in increased magnetic fields}

With future experimental studies and technological exploitation in mind, we have also explored the orientational dependence of the relative anisotropy for $\left[\mathrm{F}^{\cdot-} \mathrm{W}^{*+}\right]$ in elevated magnetic fields of intensity $1 \mathrm{mT}$ and $5 \mathrm{mT}$. Under these artificial conditions, the relative anisotropy can be substantial: We find a maximal anisotropy of $\Gamma_{\mathrm{S}}=9.9 \%$ and $4.2 \%$ for $B_{0}=1 \mathrm{mT}$ and $5 \mathrm{mT}$ respectively. Interestingly, the anisotropy is larger at $1 \mathrm{mT}$ than at $5 \mathrm{mT}$. The heat maps of the orientational dependence are summarized in Figures S7 and S8 in the SI. For $B_{0}=1 \mathrm{mT}$, the anisotropy peaks for $\beta \sim 90^{\circ}$, thereby revealing an optimal relative orientation that is practically reversed from that found at $50 \mu \mathrm{T}$. For $5 \mathrm{mT}$, the optimum is again realized for $\beta \sim 0^{\circ}$ or $\beta \sim 180^{\circ}$. Furthermore, for both $1 \mathrm{mT}$ and $5 \mathrm{mT}$, the spread of anisotropies brought about by different relative orientations is significantly smaller than that found in the geomagnetic field. For example, for $B_{0}=1 \mathrm{mT}$, the minimal relative anisotropy amounts to as much as $8.0 \%$, which is only $20 \%$ smaller than the maximal anisotropy at this field intensity. In comparison, for $B_{0}=50 \mu \mathrm{T}$, the minimal and maximal value are apart by a factor of 3 .

\section{Discussion}

\section{The model and its limitations}

For magnetoreception as an evolutionary derived trait, a high level of inherent optimization can be assumed. This suggests that vital magnetoreceptive phenotypes, such as the magnetic compass of migratory birds have evolved to maximize the sensitivity of the process. If we accept this paradigm, we may hope to reveal aspects of the biological mechanism by maximizing the predicted anisotropy in suitable models. Clearly, tenable insights will rely on the adequacy of these models, which should cater for the complexity as found in realistic systems. In particular, this requires that simulations consider a realistic number of hyperfine coupled nuclei and adequate parameters. As the Hilbert space dimension grows geometrically with the number of nuclear spins, a rigorous model quickly becomes unpractical. This necessitates simplifying assumptions such as those employed here. The current approach facilitates the fast evaluation of all relative orientations for complex spin systems by assuming the absence of inter-radical interactions, as well as identical lifetimes for the singlet and triplet state. While it is clear that these assumptions do not apply in reality, deviations from this ideal are considered small enough to avoid qualitatively different results [39,66]. As a consequence, this approximate approach has been widely employed to calculate reaction yields $[37,39,58,59,78]$. Note, however, that this does not necessarily apply to other spectroscopic techniques such as solidstate CIDNP, which requires the presence of additional, symmetry-breaking interactions to account for the accumulation of nuclear spin polarisation $[79,80]$. In general, when considering unequal reaction rate constants, it is deemed adequate, except when the triplet and singlet channels are characterized by vastly (by an order of magnitude or more) different rates. While such scenarios have been considered, they appear to be rather exotic [41]. The effects of inter-radical interactions could be small in the actual sensor [65]. We have also assumed that the spin relaxation rates are smaller than the reaction rates, while detailed simulations of the relaxation behavior suggest that they might be comparable [37]. Again, this is not expected to change the conclusions, but one needs to keep in mind that in reality the effects will likely be smaller. Detailed discussions of the assumptions made and their consequences can be seen in [39] and more recently in [66]. Semiclassical approximations have been suggested as alternative means to approach large spin systems for symmetric and asymmetric reactivity $[57,81,82]$. However, while these formulations do not suffer from the large dimensionality issues, they could overlook truly quantum effects [38]. Furthermore, for the task of evaluating many relative orientations, the approach here is in fact preferred. It does not require the 
repeated Monte Carlo sampling of the system trajectories, but can assess different orientations quickly, through the single pre-calculation of $\mathbf{X}_{l . m}\left(\vec{B}_{0}, \mathbf{R}^{T} \vec{B}_{0}\right)$.

\section{Orientational magnetosensitivity in $\left[\mathrm{F}^{\bullet-} \mathrm{W}^{\bullet+}\right]$}

The arguably most popular and currently best developed model of the cryptochrome compass assumes a magnetosensitive radical pair, comprising of a flavin anion radical and a tryptophan radical cation [4]. For this system, realistic, large-scale model calculations have only been made available for selected relative orientations of the radicals, i.e. those found for $W_{c}$ in $D m C r y ~[38,39]$ and AtCry [37]. Surprisingly, only modest singlet yield anisotropies have been found for these configurations, considering the remarkable performance of the avian compass. This raises the question, whether this is an unfortunate consequence of the relative orientation of the radical sites, which could be different in the currently unidentified magnetic sensor protein in its native environment, or perhaps, an intrinsic deficit of the $\left[\mathrm{F}^{\cdot-} \mathrm{W}^{\bullet+}\right]$ system. Our approach allows this question to be systematically addressed for radical pairs with a realistic hyperfine structure for the first time.

For our model of $\left[\mathrm{F}^{--} \mathrm{W}^{*+}\right]$ at $50 \mu \mathrm{T}$, we find relative anisotropies ranging from $0.09 \%$ to $0.27 \%$ depending on the orientation of the two radicals. Under the same condition, an anisotropy of $0.18 \%$ is found for $W_{c}$ in DmCry. Thus, in principle, the compass sensitivity could be enhanced relative to that of $W_{c}$ by a factor of 1.5 . While this is a fair improvement, the upper limit of $0.27 \%$ is still small compared to alternative models such as the hypothetical $\left[\mathrm{F}^{{ }^{-}} \mathrm{Z}^{\circ}\right]\left(\Gamma_{\mathrm{s}}=47.8 \%\right)$ or the $\left[\mathrm{F}^{{ }^{-}-}\right.$Asc $\left.{ }^{\circ-}\right]$ radical pair, for which the flavin radical is paired with the ascorbyl radical anion $\left(\Gamma_{s}=26.7 \%\right)$. While this is true, one must bear in mind that no clear-cut experimental evidence has so far been realized for any radical pair other than $\left[\mathrm{F}^{\cdot-} \mathrm{W}^{\bullet+}\right]$ in isolated cryptochromes under well-defined experimental conditions [14-16]. Superoxide has been suggested to act as $Z^{\circ}$, however, its physical properties are unlikely to comply with the requirement of slow spin relaxation times when compared to the Larmor precession frequency of an electron spin in the geomagnetic field [27]. This limitation could in principle be overcome by a three-spin system subject to the chemical Zeno effect $[28,29]$. Yet, this is an entirely speculative hypothesis that at the moment lacks even provisional experimental support and is also beyond the method employed here. As for $\left[\mathrm{F}^{\cdot-}\right.$ Asc $\left.{ }^{\cdot-}\right]$, a recent study could not identify dedicated interaction sites for the binding of $\mathrm{AscH}^{-}$to cryptochrome, which would be required to facilitate the efficient formation of the radical pair from the initially formed $\left[\mathrm{F}^{\cdot-} \mathrm{W}^{\cdot+}\right]$ prior to spin relaxation [44].

With respect to the tryptophan residues available in DmCry, the best realized orientation is that of W353, which gave rise to a relative anisotropy of $0.22 \%$ (see Table S4 in the Supporting Information). Although hypothetical, it is still interesting to note that alternative electron transfer pathways have often been observed in cryptochromes [54-56]. Thus, alternative tryptophans should not be excluded a priori. However, no experimental evidence for the involvement of W353 or an equivalent site seems to have been documented. More generally, for $B_{0}=50 \mu \mathrm{T}$, large anisotropies appear to tentatively relate to larger mean singlet yields $\left\langle\Phi_{s}\right\rangle$. In particular, when combining all tested relative orientations, for $\left\langle\Phi_{s}\right\rangle>0.256$ the relative anisotropy grows approximately linearly with $\left\langle\Phi_{s}\right\rangle$. This suggests that the large compass sensitivity observed here is tantamount to slower relaxation of the singlet population to it statistical expectation of $1 / 4$. This is not surprising insofar, as weak magnetic fields can only give rise to discernible effects on radical pairs if the spin systems are out of equilibrium.

We are currently unaware what magnitudes of MFEs are necessary to constitute a compass sense. Despite this, if we assume for the moment that the directionality of the MFEs in $\left[\mathrm{F}^{{ }^{--}} \mathrm{W}^{*+}\right]$ is sufficient, the following observations can be made: Based on the currently available structures of cryptochromes with a tryptophan tetrad, $W_{C}$ appears to be more apt to induce directional MFEs than $W_{D}, W_{A}$ or $W_{B}$. 
This is reassuring insofar as MFEs on isolated cryptochromes have only been observed for the radical pair involving $W_{C}$. The pair with $W_{D}$ appears to be separated by too large a distance to facilitate MFEs, i.e. its slow spin selective charge recombination reaction cannot compete with decoherence processes, whereby any MFEs are likely abolished $[14,83,84]$. For the close radical pairs with $W_{A}$ and $W_{B}$, on the other hand, the large exchange interaction and short lifetimes are deemed to suppress the magnetosensitivity [65]. It is also remarkable that the exceptional directional sensitivity of $W_{c}$ appears to be peculiar to cryptochromes with a tryptophan tetrad. For the Arabidopsis cryptochrome 1 , on the other hand, $W_{c}$ only corresponds to modest orientational sensitivity, comparable to the predictions for $W_{A}$ and $W_{B}$. As a directional response to magnetic fields does not fulfil a known function in plants, this could be interpreted as an outcome of the lack of a corresponding evolutionary incentive. Note, however, that while $W_{c}$ is strong in cryptochromes with a tetrad, the orientation of $W_{c}$ in the homology model for the avian cryptochrome $4[75,76]$ is less optimal than that extracted from the crystal structure of the Drosophila cryptochrome. This observation could inspire a variety of interpretations: the homology model or the current approach may have limited predictive power, another driver must be considered, anisotropy is an inappropriate measure of sensitivity or that a different radical pair is at play, to name but a few. Much additional theoretical and experimental research will be required to start addressing these points.

Various measures have been employed to assess directional MFEs. Here, we used the relative and the absolute anisotropy as defined in eq. (19). As the signal transduction and amplification mechanisms are unknown, it is unclear which is more indicative. As far as the relative orientation is concerned, $\Delta_{S}$ and $\Gamma_{s}$ provide essentially conforming conclusions and this distinction does not appear to be relevant. However, two recent works argue that the spikiness of the anisotropy pattern might be more relevant than the absolute size of the effect, as it could provide a pathway to, and rationale for, the remarkable acuity of the avian compass $[38,41]$. Solov'yov and co-workers have suggested an alternative measure, referred to as optimality, which quantifies spikiness [41]. Defined here by

$$
\text { optimality }=\Gamma_{s}\left(1-2 \frac{\left\langle\Phi_{s}\right\rangle-\min _{\theta, \phi} \Phi_{s}\left(\vec{B}_{0}(\theta, \phi)\right)}{\Delta_{s}}\right),
$$

this measure is only non-zero for spiky profiles, i.e. those for which the mean yield $\left\langle\Phi_{s}\right\rangle$ deviates from the average of the minimal and maximal yield, $\frac{1}{2}\left(\min _{\theta, \phi} \Phi_{s}\left(\vec{B}_{0}(\theta, \phi)+\max _{\theta, \phi} \Phi_{s}\left(\vec{B}_{0}(\theta, \phi)\right)\right.\right.$ (the original publication used a slightly different definition for $\Gamma_{s}$ ). Analyzing our data with respect to eq. (20), we have to conclude that in $\left[\mathrm{F}^{\cdot-} \mathrm{W}^{\cdot+}\right]$ anisotropy and optimality are mutually exclusive (see Figure $\mathrm{S9}$ ). In particular, we find that optimality peaks for $\beta \sim 90^{\circ}$, a region for which the anisotropy is always low. Conversely, large anisotropies appear to favor simple shapes. It is also noteworthy that the dependence of optimality on $\gamma$ and, in particular, $\alpha$ is more pronounced than for the anisotropy. The anti-correlation of optimality and anisotropy is an interesting finding in view of $W_{c}$ 's putative role in magnetoreception, as this system produces the worst optimality among the tryptophans of the tryptophan tetrad. If $W_{C}$ indeed constitutes the magnetosensitive radical pair, this could infer that optimality is either an inferior metric, or perhaps that a compromise between these two metrics is necessary for optimizing the compass. On the other hand, if optimality is key, the radical pair involving $W_{D}$ would be almost optimum with respect to $\beta$ and $\gamma$, as is seen from the location of the top relative orientation for optimality in Figure $S 9$ (maximal optimality realized for $\alpha=-27^{\circ}, \beta=93^{\circ}, \gamma=0^{\circ}$ ). This would, however require that the charge recombination proceeds on a timescale comparable to the spin evolution, which has been questioned $[14,83,84]$. In any case, it is interesting to observe that anisotropy and optimality as mediators of compass sensitivity favor orthogonally different $\left[\mathrm{F}^{\circ-} \mathrm{W}^{\bullet+}\right]$ 
radical pairs. This suggests that a biologically motivated assessment of the best metric, which is unfortunately not yet available, could guide the search for the true magnetoreceptor.

In addition to impacting upon the attainable MFEs, the relative orientation of the radicals is also expected to influence the electron transfer rates, because it modulates the electronic coupling of the involved diabatic states [85]. However, this does not necessarily allow direct conclusions regarding the observed electron transfer rates, as the relevant tunnelling processes are often controlled by coupling fluctuations instead of the average coupling, in particular, if symmetry implied a weak coupling at the average geometry [86]. Indeed, a recent study of charge migration within the tryptophan tetrad of an animal (6-4) photolyase has found no clear relationship of electronic coupling fluctuations and the relative orientations of the involved tryptophan residues, while the edge-to-edge distance was identified as decisive [83]. Thus, while orientational effects on electron transfer exist, they do not a priori limit the configurations which could practically be realized.

\section{Orientational magnetosensitivity in $\left[\mathrm{F}^{\bullet-} \mathrm{Y}^{\bullet}\right]$}

Long-lived tyrosine radicals have been observed in several studies of cryptochromes. $Y^{\bullet}$ was identified as a successor of $\mathrm{W}^{\cdot+}$ in the photo-activation of cryptochrome 1a of the garden warbler [87], cryptochrome 1 from Arabidopsis thaliana [88], and the animal-like cryptochrome from C. reinhardtii [19]. In the latter, the radicalized tyrosine (Y373) extends the protein's tryptophan triad and appears to be essential for the photo-reduction in order to yield the fully reduced $\mathrm{FAD}^{-}$[19]. A similar extension of a tryptophan tetrad is seen in the predicted structure of cryptochrome 4 from the European robin [75]. While the formation of $Y^{\bullet}$ appears commonplace in cryptochromes, no MFEs on reaction yields have so far been associated with $\mathrm{Y}^{\bullet}$-containing radical pairs. Given the larger magnetic anisotropies that we predict here, it is nonetheless tempting to speculate about this possibility. This conjecture is further fueled by the fact that spin polarized EPR spectra of photo-generated $\left[\mathrm{F}^{\circ-} \mathrm{Y}^{\circ}\right]$ have been observed in $C$. reinhardtii, thereby demonstrating that spin coherent effects are in principle feasible in these radical pairs in cryptochromes [19].

The $\left[\mathrm{F}^{\cdot-} \mathrm{Y}^{*}\right]$ radical pair offers many virtues; first and foremost, its anisotropy is predicted to markedly exceed that of $\left[\mathrm{F}^{\cdot-} \mathrm{W}^{*+}\right]$. Relative anisotropies approaching $1 \%$ have been found here, which compares favorably to the maximal anisotropy of $\left[\mathrm{F}^{\cdot-} \mathrm{W}^{*+}\right]$ of $0.27 \%$. The larger anisotropy appears to be a consequence of the weaker intrinsic anisotropy of $Y^{\bullet}$ that puts $\left[F^{\circ-} Y^{*}\right]$ closer to the ideal $\left[F^{\circ-} Z^{*}\right]$. This is also evident from the smaller anisotropy of $\left[Z^{\bullet} Y^{*}\right]$ compared to $\left[Z^{*} W^{*+}\right]$. Yet, while $\left[F^{-{ }^{-}} Y^{*}\right]$ appears to be a better choice than $\left[\mathrm{F}^{\cdot-} \mathrm{W}^{*+}\right]$, its anisotropy is still small relative to $\left[\mathrm{F}^{{ }^{--}} \mathrm{Z}^{\circ}\right]$. However, as the latter has to be viewed as a hypothetical entity, $\left[\mathrm{F}^{{ }^{-}} \mathrm{Y}^{*}\right]$ might still be the optimal radical pair assembled from redox-active residues in proteins. Secondly, unlike for $\left[\mathrm{F}^{\circ-} \mathrm{W}^{*+}\right]$, anisotropy and optimality, i.e. the spikiness of the anisotropy pattern, are correlated for $\left[\mathrm{F}^{\cdot-} \mathrm{Y}^{*}\right]$. Both measures are maximal for a relative orientation of the radicals with approximately perpendicular orientation of the aromatic ring planes. Taken together, our findings could provide a significant evolutionary driver in favor of $\left[\mathrm{F}^{{ }^{-}} \mathrm{Z}^{\circ}\right]$, which warrants further studies into this direction. Using the known crystal structure of DmCry as a template [51, 52], the residues Y317, Y319 and Y328 appear the most auspicious candidates for future studies of this kind. These residues give rise to the largest anisotropies amongst all tyrosines in DmCry, while also being located close to the flavin co-factor, thereby facilitating the necessary charge recombination reaction that could be part of alternative electron transfer pathways. For Y 319 , the adjacent $\mathrm{H} 138$ could facilitate the electron transfer, e.g. from the adjacent W413, by deprotonating the tyrosine. Indeed, there appears to be some evidence that, at least in plants, in vivo electron transfer reactions along pathways not involving the tryptophan tetrad could be relevant $[54,55]$. Much of this evidence, however, is from Cry variants where members of the triad have been mutated. The significance of these findings for the native protein is currently unclear.

Obviously, the generation of the hypothesised tyrosine-based radical pairs required that in vivo the electron transfer pathway deviated from the canonical pathway provided by the tryptophan triad/tetrad. This raises the questions of competing electron transfer pathways and non-uniform 
populations of radical pairs. In this regard, it is interesting to note that in the avian Cry4 the tryptophan tetrad ends at a tyrosine site [75]. If the magnetosensitive RP involved this tyrosine, the aforementioned question could be avoided. While attractive, this would require a different functional role of the tetrad, which would have to act as a reversible shuttle of electrons between the flavin and the tyrosine rather than a mere pathway of efficient charge separation. This would necessitate a reduced driving force of charge separation along the tetrad, which could in principle be brought about by a less polar environment at the protein surface (in comparison to the aqueous buffers used in in vitro experiments).

\section{Entanglement}

When the radical pair is formed in the singlet electronic state, the spins of its electrons are entangled. This aspect has attracted much recent attention, in part because it implies a truly quantum aspect characteristic of magnetoreception [4, 42, 43, 70, 89-91]. Yet, it is all but clear if entanglement plays an important functional role, e.g. in boosting the sensitivity or precision of the directional response to a weak magnetic field. Cautious voices have suggested this is likely not the case, as separable initial states such as unpolarized triplet states can provide comparable or even larger anisotropies [70, 89]. Noting that previous papers focusing on entanglement in magnetoreception have all employed simple model spin systems that do not reflect the large number of coupled magnetic nuclei, we have decided to once again explore this aspect. Reconstructing the electronic density operator from the spin correlation tensors, the negativity, a popular entropy monotone, can be evaluated for complex spin systems. Our analysis suggests that for the large spin systems considered here, the negativity drops to zero within nanoseconds. In the $\left[\mathrm{F}^{\bullet-} \mathrm{W}^{*+}\right]$-model we could not observe a rebirth of entanglement at any later time irrespective of the mutual orientation of the radicals (entanglement lifetime: $\sim 5 \mathrm{~ns}$; see Figure S11). Only for the hypothetical $\left[\mathrm{F}^{\bullet-} \mathrm{Z}^{*}\right]$ and $\left[\mathrm{Z}^{\bullet} \mathrm{Y}^{\bullet}\right]$, a second minor burst is observed. Despite this, entanglement within the system is short-lived. As a consequence of its ephemerality, it does not show an apparent dependence on the orientation of the external magnetic field. This implies that it cannot underpin a crucial aspect of the magnetic anisotropy for any of the models with large numbers of hyperfine-coupled nuclei. It is noteworthy that this statement also applies to $\left[\mathrm{F}^{\bullet-} \mathrm{Z}^{\bullet}\right]$, even though its hyperfine structure is dominated by the nitrogen nuclei at positions 5 and 10, which, if present alone, do sustain negativity much longer (see Figure S12 in the SI). This is remarkable insofar as the N5N10-model provides an adequate reflection as far as the singlet anisotropy is concerned, i.e. the additional nuclear spins in more extensive models hardly alter the singlet anisotropy. Based on these observations, we conclude that the postulated roles of entanglement are likely an upshot of oversimplified spin systems $[42,43,91]$. Additionally, we also highlight that all eigenvalues of $\hat{\rho}_{e l}^{T_{A}}$ are found to be smaller than (or, at $t=0$, equal to) $1 / 2$ for the singlet-born radical pair. As discussed above, this implies that the negativity of the analogue triplet-born radical pair is not only zero initially, but

zero throughout. Nonetheless, as $\Phi_{s}(T-$-born $)=\frac{1}{3}-\frac{1}{3} \Phi_{s}(S$-born) the anisotropy is expected to be of the same order of magnitude (assuming that the singlet yield approaches $1 / 4$, which is the case for longlived radical pairs, the anisotropy is only smaller by a factor of $1 / 3$ for the triplet-born pair). This once again corroborates the argument that entanglement is futile given that separable states provide comparable effects [4]. While we cannot exclude the possibility that entanglement plays an important role in other scenarios than those studied here, our findings suggest that it is likely irrelevant for the hyperfine-dominated MFE of radical pairs of realistic complexity. 


\section{Conclusions}

We have suggested a method to efficiently evaluate the orientational dependence of magnetic anisotropy parameters for radical pairs with negligible inter-radical coupling and equal reactivity in the singlet and triplet manifold. This approach has been applied to models of radical pairs pertinent to the radical pair hypothesis of the avian compass, whereby a realistic number of hyperfine interactions could be considered. While the identity of the actual magnetosensitive radical pair in cryptochromes under in vivo conditions remains unknown, here, we have focused on radical pairs comprising the flavin radical anion and either the tryptophan radical cation or the tyrosine neutral radical.

By systematically probing all relative orientations of the radicals in the pair, we have identified the optimal relative orientations with respect to the anisotropy of the singlet yield, a measure of the sensitivity of the compass, and optimality, a measure of its precision. We find that for $\left[\mathrm{F}^{\bullet-} \mathrm{W}^{\bullet+}\right]$ in the geomagnetic field, these two parameters are anti-correlated and, thus, both metrics cannot be simultaneously maximized. The anisotropy is largest for parallel orientations of the aromatic planes of the radicals; maximal optimality results for perpendicular orientations. Among the tryptophans of the tryptophan tetrad for the Drosophila cryptochrome, $W_{c}$ exhibits the largest anisotropy (and, thus, necessarily unfavorable optimality). The optimum relative orientation (relative anisotropy: $0.27 \%$ ) gave rise to $\sim 50 \%$ increase in anisotropy with respect to $W_{c}$ 's orientation within the crystal structure. For $W_{D}$, nearly maximal optimality was realized (provided that the charge recombination reaction was not too small to abolish the MFE). Conversely, its anisotropy was minute. It seems likely that natural selection has provided the necessary pressure to ensure that in migratory birds, a relative orientation is realized for optimal navigational performance. For an $\left[\mathrm{F}^{\cdot-} \mathrm{W}^{*+}\right]$-based compass, this would necessitate compromising on anisotropy and optimality.

Radical pairs containing tyrosine outperform $\left[\mathrm{F}^{\bullet-} \mathrm{W}^{\bullet+}\right]$ for most relative orientations. An optimal anisotropy of $0.96 \%$ can be realized. Furthermore, optimality and anisotropy are correlated and can be simultaneously maximized for perpendicular orientations of the radical aromatic planes.

In general, we conclude that no relative orientation of both $\left[\mathrm{F}^{\cdot-} \mathrm{W}^{*+}\right]$ and $\left[\mathrm{F}^{\cdot-} \mathrm{Y}^{*}\right]$ can reinstate effect sizes comparable to the hypothetical $\left[\mathrm{F}^{\bullet-} \mathrm{Z}^{+}\right]$-model. While nothing is known about the required anisotropy of the primary reaction to sustain the compass, this corroborates our impression that alternative models such as those recently suggested based on three-radical effects $[28,29,92]$ could be at play in the presumably highly-optimized and exquisitely sensitive avian compass. This supposition is further substantiated by the recent claims of magnetoreception in the dark, which seems to conflict with the radical pair paradigm, as it implicates swiftly relaxing species such as superoxide [7, 23]. Alternatively, [ $\left.\mathrm{F}^{\cdot-} \mathrm{Y}^{*}\right]$-based systems appear better versed than their tryptophancontaining counterparts. Altogether, this leaves much room for future studies both, of the theoretical underpinnings and the functional realization of magnetoreception in cryptochromes.

For applications of $\left[\mathrm{F}^{\bullet-} \mathrm{W}^{\bullet+}\right]$ at elevated magnetic fields, substantial anisotropies on the order of $10 \%$ are possible at $1 \mathrm{mT}$. Furthermore, the orientational spread of the performance measures is smaller, suggesting that the relative orientation is of minor concern. Yet, maximal effects are expected for perpendicular orientations of the radical planes, contrary to what optimized the effect in the geomagnetic field.

The fast decay of negativity within both $\left[\mathrm{F}^{\cdot-} \mathrm{W}^{\bullet+}\right]$ and $\left[\mathrm{F}^{\circ-} \mathrm{Z}^{*}\right]$ radical pairs involving a realistic number of hyperfine interactions suggests an inconsequential role of electronic entanglement within the Radical Pair Mechanism when applied to the avian compass system. This could imply that, unless prominently stated in the literature on quantum biology, coherence, or the decay of coherence, might be the sole quantum property of relevance to magnetoreception. 


\section{Appendix}

\section{Proof of eq. (9):}

For notational simplicity, we suppress the explicit dependence of the spin correlation tensor on the identity of the radical, $i$. Hence, the $\alpha, \beta$-matrix element of $\mathbf{T}(t)$ is

$$
T_{\alpha, \beta}(t)=\frac{1}{z} \operatorname{Tr}\left[\hat{S}_{\alpha} e^{+i \hat{H} t} \hat{S}_{\beta} e^{-i \hat{H} t}\right]
$$

We shall initially explore the behavior of $\mathbf{T}(t)$ under rotation,

$$
\mathbf{T}^{\prime}(t)=\mathbf{R} \mathbf{T}(t) \mathbf{R}^{-1},
$$

for a rotation around the $z$-axis, i.e. for the rotation described by the rotation matrix [93]

$$
\mathbf{R}=\mathbf{R}_{z}(\alpha)=\left(\begin{array}{ccc}
\cos \alpha & -\sin \alpha & 0 \\
\sin \alpha & \cos \alpha & 0 \\
0 & 0 & 1
\end{array}\right)
$$

Using the properties of the spin operators under rotation,

$$
\cos (\alpha) \hat{S}_{x} \pm \sin (\alpha) \hat{S}_{y}=e^{-i \alpha \hat{S}_{z}} \hat{S}_{x, y} e^{+i \alpha \hat{S}_{z}}
$$

it is straight-forward to show that the elements of the rotated tensor are given by

$$
T_{\alpha, \beta}^{\prime}(t)=\frac{1}{Z} \operatorname{Tr}\left[e^{+i \alpha \hat{S}_{2}} \hat{S}_{\alpha} e^{-i \alpha \hat{S}_{z}} e^{+i \hat{H} t} e^{+i \alpha \hat{S}_{z}} \hat{S}_{\beta} e^{-i \alpha \hat{S}_{z}} e^{-i \hat{H} t}\right]
$$

Inserting $1=\prod_{j} e^{+i \alpha l_{j}} e^{-i \alpha l_{j}}$ and making use of the commutativity of electron and nuclear spin operators and the constant of the trace of products of operators to cyclic permutation, eq. (25) can be rewritten as

$$
T_{\alpha, \beta}^{\prime}(t)=\frac{1}{z} \operatorname{Tr}\left[\hat{S}_{\alpha} e^{+i \hat{H}^{\prime} t} \hat{S}_{\beta} e^{-i \hat{H}^{\prime} t}\right],
$$

where

$$
\hat{H}^{\prime}=e^{-i \alpha \hat{s}_{2}} \prod_{j} e^{-i \alpha \hat{l}_{j, 2}} \hat{H} e^{+i \alpha \hat{S}_{z}} \prod_{1} e^{+i \alpha \hat{l_{1,2}}}
$$

Using eq. (24) once more, this time in the reverse direction, allows to re-express $\hat{H}^{\prime}$ in terms of the rotated hyperfine tensors and field by realizing that

$$
\begin{aligned}
\hat{H}^{\prime} & =\sum_{j}\left(\mathbf{R}^{T} \hat{\mathbf{S}}\right) \cdot \mathbf{A}_{j} \cdot\left(\mathbf{R}^{T} \hat{\mathbf{l}}_{j}\right)+\vec{\omega}_{0} \cdot\left(\mathbf{R}^{T} \hat{\mathbf{S}}\right) \\
& =\sum_{j} \hat{\mathbf{S}} \cdot \mathbf{R} \mathbf{A}_{j} \mathbf{R}^{T} \cdot \hat{\mathbf{l}}_{j}+\left(\mathbf{R} \vec{\omega}_{0}\right) \cdot \hat{\mathbf{S}}
\end{aligned}
$$

and, thus,

$$
\mathbf{R} \mathbf{T}\left(t ; \vec{B}_{0},\left\{\mathbf{A}_{j}\right\}\right) \mathbf{R}^{-1}=\mathbf{T}\left(t ; \mathbf{R} \vec{B}_{0},\left\{\mathbf{R A}_{j} \mathbf{R}^{-1}\right\}\right) .
$$


An analogous derivation shows that eq. (29) also applies for a rotation about the $x$-axis. As any rotation can be decomposed into consecutive rotations about the $z-, x-$, and $z$-axis [93], this proves eq. (29) for general rotations, R . Eq. (9) follows from eq. (29) by substituting $\vec{B}_{0}$ by $\mathbf{R}^{-1} \vec{B}_{0}$.

\section{Euler angle convention}

We make use of active rotations and parametrize rotations by the $z x z$-convention (also see Figure S2 in the Supporting Information). In order to avoid ambiguity, we specify the rotation matrix in terms of Euler angles:

$$
\mathbf{R}(\alpha, \beta, \gamma)=\left(\begin{array}{ccc}
c_{\alpha} c_{\gamma}-c_{\beta} s_{\alpha} s_{\gamma} & -c_{\beta} c_{\gamma} s_{\alpha}-c_{\alpha} s_{\gamma} & s_{\alpha} s_{\beta} \\
c_{\gamma} s_{\alpha}+c_{\alpha} c_{\beta} s_{\gamma} & c_{\alpha} c_{\beta} c_{\gamma}-s_{\alpha} s_{\gamma} & -c_{\alpha} s_{\beta} \\
s_{\beta} s_{\gamma} & c_{\gamma} s_{\beta} & c_{\beta}
\end{array}\right) .
$$

Here, $c_{a}=\cos (a)$ and $s_{a}=\sin (a)$.

\section{Supplementary Material}

See the supplementary material for a compilation of the hyperfine tensors used in this study, information on the choice representative sidechain dihedral angles, the definition of the molecular axis systems, and additional data on the MFEs, including anisotropy plots and the time-dependence of the entanglement.

\section{Acknowledgments}

We thank EPSRC (Grant No. EP/R021058/1) for financial support and NVIDIA for providing a Titan Xp GPU through their GPU Grant Program.

\section{References}

[1] H. Mouritsen, Long-distance navigation and magnetoreception in migratory animals, Nature 558 (2018) 50-59.

[2] G.C. Nordmann, T. Hochstoeger, D.A. Keays, Unsolved mysteries: Magnetoreception-a sense without a receptor, PLoS Biol. 15 (2017).

[3] R. Wiltschko, W. Wiltschko, Magnetic Orientation in Animals, Springer, Berlin, New York, 1995.

[4] P.J. Hore, H. Mouritsen, The radical-pair mechanism of magnetoreception, Annu. Rev. Biophys. 45 (2016) 299-344.

[5] K. Schulten, C.E. Swenberg, A. Weller, A biomagnetic sensory mechanism based on magnetic field modulated coherent electron spin motion, Z. Phys. Chem. 111 (1978) 1-5.

[6] T. Ritz, S. Adem, K. Schulten, A model for photoreceptor-based magnetoreception in birds, Biophys. J. 78 (2000) 707-718.

[7] M. Pooam, L.D. Arthaut, D. Burdick, J. Link, C.F. Martino, M. Ahmad, Magnetic sensitivity mediated by the Arabidopsis blue-light receptor cryptochrome occurs during flavin reoxidation in the dark, Planta (2018).

[8] M. Ahmad, Photocycle and signaling mechanisms of plant cryptochromes, Curr. Opin. Plant Biol. 33 (2016) 108-115.

[9] M.E. Maffei, Magnetic field effects on plant growth, development, and evolution, Frontiers in Plant Science 5 (2014). 
[10] J.A.T. da Silva, J. Dobranszki, Magnetic fields: how is plant growth and development impacted?, Protoplasma 253 (2016) 231-248.

[11] R.J. Gegear, A. Casselman, S. Waddell, S.M. Reppert, Cryptochrome mediates light-dependent magnetosensitivity in Drosophila, Nature 454 (2008) 1014-U1061.

[12] O. Bazalova, M. Kvicalova, T. Valkova, P. Slaby, P. Bartos, R. Netusil, K. Tomanova, P. Braeunig, H.J. Lee, I. Sauman, M. Damulewicz, J. Provaznik, R. Pokorny, D. Dolezel, M. Vacha, Cryptochrome 2 mediates directional magnetoreception in cockroaches, Proc. Natl. Acad. Sci. U. S. A. 113 (2016) 1660-1665.

[13] J.B. Phillips, S.C. Borland, Behavioral evidence for use of a light-dependent magnetoreception mechanism by a vertebrate, Nature 359 (1992) 142-144.

[14] D.M.W. Sheppard, J. Li, K.B. Henbest, S.R.T. Neil, K. Maeda, J. Storey, E. Schleicher, T. Biskup, R. Rodriguez, S. Weber, P.J. Hore, C.R. Timmel, S.R. Mackenzie, Millitesla magnetic field effects on the photocycle of an animal cryptochrome, Sci. Rep. 7 (2017) 42228.

[15] K. Maeda, A.J. Robinson, K.B. Henbest, H.J. Hogben, T. Biskup, M. Ahmad, E. Schleicher, S. Weber, C.R. Timmel, P.J. Hore, Magnetically sensitive light-induced reactions in cryptochrome are consistent with its proposed role as a magnetoreceptor, Proc. Natl. Acad. Sci. U. S. A. 109 (2012) 4774-4779.

[16] D.R. Kattnig, E.W. Evans, V. Dejean, C.A. Dodson, M.I. Wallace, S.R. Mackenzie, C.R. Timmel, P.J. Hore, Chemical amplification of magnetic field effects relevant to avian magnetoreception, Nat. Chem. 8 (2016) 384-391.

[17] Z. Zeng, J.J. Wei, Y.Y. Liu, W.D. Zhang, T. Mabe, Magnetoreception of photoactivated cryptochrome 1 in electrochemistry and electron transfer, Acs Omega 3 (2018) 4752-4759.

[18] D. Nohr, B. Paulus, R. Rodriguez, A. Okafuji, R. Bittl, E. Schleicher, S. Weber, Determination of radical-radical distances in light-active proteins and their implication for biological magnetoreception, Angew. Chem. Int. Ed. 56 (2017) 8550-8554.

[19] D. Nohr, S. Franz, R. Rodriguez, B. Paulus, L.O. Essen, S. Weber, E. Schleicher, Extended electrontransfer in animal cryptochromes mediated by a tetrad of aromatic amino acids, Biophys. J. 111 (2016) 301-311.

[20] G.L. Closs, L.E. Closs, Induced Dynamic Nuclear Spin Polarization in Photoreductions of Benzophenone by Toluene and Ethylbenzene, J. Am. Chem. Soc. 91 (1969) 4550-\&.

[21] G.L. Closs, A Mechanism Explaining Nuclear Spin Polarizations in Radical Combination Reactions, J. Am. Chem. Soc. 91 (1969) 4552-\&.

[22] R. Kaptein, J.L. Oosterhoff, Chemically induced dynamic nuclear polarization II - (Relation with anomalous ESR spectra), Chem. Phys. Lett. 4 (1969) 195-197.

[23] R. Wiltschko, M. Ahmad, C. Nießner, D. Gehring, W. Wiltschko, Light-dependent magnetoreception in birds: The crucial step occurs in the dark, J. R. Soc., Interface 13 (2016) 20151010.

[24] C. Nießner, S. Denzau, L. Peichl, W. Wiltschko, R. Wiltschko, Magnetoreception in birds: I. Immunohistochemical studies concerning the cryptochrome cycle, J. Exp. Biol. 217 (2014) 42214224.

[25] R. Wiltschko, D. Gehring, S. Denzau, C. Nießner, W. Wiltschko, Magnetoreception in birds: II. Behavioural experiments concerning the cryptochrome cycle, J. Exp. Biol. 217 (2014) 4225-4228.

[26] I.A. Solov'yov, K. Schulten, Magnetoreception through cryptochrome may involve superoxide, Biophys. J. 96 (2009) 4804-4813.

[27] H.J. Hogben, O. Efimova, N. Wagner-Rundell, C.R. Timmel, P.J. Hore, Possible involvement of superoxide and dioxygen with cryptochrome in avian magnetoreception: Origin of Zeeman resonances observed by in vivo EPR spectroscopy, Chem. Phys. Lett. 480 (2009) 118-122.

[28] D.R. Kattnig, P.J. Hore, The sensitivity of a radical pair compass magnetoreceptor can be significantly amplified by radical scavengers, Sci. Rep. 7 (2017) 11640.

[29] D.R. Kattnig, Radical-pair-based magnetoreception amplified by radical scavenging: Resilience to spin relaxation, J. Phys. Chem. B 121 (2017) 10215-10227. 
[30] J.C.S. Lau, N. Wagner-Rundell, C.T. Rodgers, N.J.B. Green, P.J. Hore, Effects of disorder and motion in a radical pair magnetoreceptor, J. R. Soc., Interface 7 (2010) S257-S264.

[31] I.A. Solov'yov, H. Mouritsen, K. Schulten, Acuity of a cryptochrome and vision-based magnetoreception system in birds, Biophys. J. 99 (2010) 40-49.

[32] E. Hill, T. Ritz, Can disordered radical pair systems provide a basis for a magnetic compass in animals?, J. R. Soc., Interface 7 (2010) S265-S271.

[33] T.J. Zwang, E.C.M. Tse, D.P. Zhong, J.K. Barton, A compass at weak magnetic fields using thymine dimer repair, Acs Central Science 4 (2018) 405-412.

[34] P.J. Hore, A DNA-based magnetic sensor, Acs Central Science 4 (2018) 318-320.

[35] S. Worster, D.R. Kattnig, P.J. Hore, Spin relaxation of radicals in cryptochrome and its role in avian magnetoreception, J. Chem. Phys. 145 (2016) 035104.

[36] D.R. Kattnig, J.K. Sowa, I.A. Solov'yov, P.J. Hore, Electron spin relaxation can enhance the performance of a cryptochrome-based magnetic compass sensor, New J. Phys. 18 (2016) 063007. [37] D.R. Kattnig, I.A. Solov'yov, P.J. Hore, Electron spin relaxation in cryptochrome-based magnetoreception, Phys. Chem. Chem. Phys. 18 (2016) 12443-12456.

[38] H.G. Hiscock, S. Worster, D.R. Kattnig, C. Steers, Y. Jin, D.E. Manolopoulos, H. Mouritsen, P.J. Hore, The quantum needle of the avian magnetic compass, Proc. Natl. Acad. Sci. U. S. A. 113 (2016) 4634-4639.

[39] A.A. Lee, J.C.S. Lau, H.J. Hogben, T. Biskup, D.R. Kattnig, P.J. Hore, Alternative radical pairs for cryptochrome-based magnetoreception, J. R. Soc., Interface 11 (2014) 20131063.

[40] M. Procopio, T. Ritz, Inhomogeneous ensembles of radical pairs in chemical compasses, Sci. Rep. 6 (2016).

[41] J.B. Pedersen, C. Nielsen, I.A. Solov'yov, Multiscale description of avian migration: from chemical compass to behaviour modeling, Sci. Rep. 6 (2016) 36709.

[42] E.M. Gauger, E. Rieper, J.J.L. Morton, S.C. Benjamin, V. Vedral, Sustained quantum coherence and entanglement in the avian compass, Phys. Rev. Lett. 106 (2011) 040503.

[43] Y.T. Zhang, G.P. Berman, S. Kais, The radical pair mechanism and the avian chemical compass: Quantum coherence and entanglement, Int. J. Quantum Chem. 115 (2015) 1327-1341.

[44] C. Nielsen, D.R. Kattnig, E. Sjulstok, P.J. Hore, I.A. Solov'yov, Ascorbic acid may not be involved in cryptochrome-based magnetoreception, J. R. Soc., Interface 14 (2017) 20170657.

[45] N. Lambert, Y.N. Chen, Y.C. Cheng, C.M. Li, G.Y. Chen, F. Nori, Quantum biology, Nature Physics 9 (2013) 10-18.

[46] S.F. Huelga, M.B. Plenio, Vibrations, quanta and biology, Contemporary Physics 54 (2013) 181207.

[47] A. Marais, B. Adams, A.K. Ringsmuth, M. Ferretti, J.M. Gruber, R. Hendrikx, M. Schuld, S.L.

Smith, I. Sinayskiy, T.P.J. Kruger, F. Petruccione, R. van Grondelle, The future of quantum biology, J.

R. Soc., Interface 15 (2018).

[48] S. Oldemeyer, S. Franz, S. Wenzel, L.O. Essen, M. Mittag, T. Kottke, Essential role of an unusually long-lived tyrosyl radical in the response to red light of the animal-like cryptochrome acry, J. Biol. Chem. 291 (2016) 14062-14071.

[49] C. Thöing, S. Oldemeyer, T. Kottke, Microsecond deprotonation of aspartic acid and response of the alpha/beta subdomain precede c-terminal signaling in the blue light sensor plant cryptochrome, J. Am. Chem. Soc. 137 (2015) 5990-5999.

[50] T. Biskup, B. Paulus, A. Okafuji, K. Hitomi, E.D. Getzoff, S. Weber, E. Schleicher, Variable electron transfer pathways in an amphibian cryptochrome: Tryptophan versus tyrosine-based radical pairs, J.

Biol. Chem. 288 (2013) 9249-9260.

[51] B.D. Zoltowski, A.T. Vaidya, D. Top, J. Widom, M.W. Young, B.R. Crane, Structure of full-length Drosophila cryptochrome, Nature 480 (2011) 396-399.

[52] C. Levy, B.D. Zoltowski, A.R. Jones, A.T. Vaidya, D. Top, J. Widom, M.W. Young, N.S. Scrutton, B.R. Crane, D. Leys, Updated structure of Drosophila cryptochrome, Nature 495 (2013) E3-E4. 
[53] C.A. Brautigam, B.S. Smith, Z.Q. Ma, M. Palnitkar, D.R. Tomchick, M. Machius, J. Deisenhofer, Structure of the photolyase-like domain of cryptochrome 1 from Arabidopsis thaliana, Proc. Natl. Acad. Sci. U. S. A. 101 (2004) 12142-12147.

[54] M. El-Esawi, A. Glascoe, D. Engle, T. Ritz, J. Link, M. Ahmad, Cellular metabolites modulate in vivo signaling of Arabidopsis cryptochrome-1, Plant Signaling \& Behavior 10 (2015).

[55] C. Engelhard, X.C. Wang, D. Robles, J. Moldt, L.O. Essen, A. Batschauer, R. Bittl, M. Ahmad, Cellular metabolites enhance the light sensitivity of Arabidopsis cryptochrome through alternate electron transfer pathways, Plant Cell 26 (2014) 4519-4531.

[56] T. Biskup, K. Hitomi, E.D. Getzoff, S. Krapf, T. Koslowski, E. Schleicher, S. Weber, Unexpected electron transfer in cryptochrome identified by time-resolved epr spectroscopy, Angew. Chem. Int. Ed. 50 (2011) 12647-12651.

[57] K. Schulten, P.G. Wolynes, Semiclassical description of electron spin motion in radicals including the effect of electron hopping, The Journal of Chemical Physics 68 (1978) 3292-3297.

[58] N.N. Lukzen, D.R. Kattnig, G. Grampp, The effect of signs of hyperfine coupling constant on MARY spectra affected by degenerate electron exchange, Chem. Phys. Lett. 413 (2005) 118-122. [59] D.R. Kattnig, A. Rosspeintner, G. Grampp, Fully reversible interconversion between locally excited fluorophore, exciplex, and radical ion pair demonstrated by a new magnetic field effect, Angew. Chem. Int. Ed. 47 (2008) 960-962.

[60] L. Kowalik, J.K. Chen, Illuminating developmental biology through photochemistry, Nat. Chem. Biol. 13 (2017) 587-598.

[61] C.N.G. Giachello, N.S. Scrutton, A.R. Jones, R.A. Baines, Magnetic fields modulate blue-lightdependent regulation of neuronal firing by cryptochrome, J. Neurosci. 36 (2016) 10742-10749.

[62] S. Paul, A.S. Kiryutin, J.P. Guo, K.L. Ivanov, J. Matysik, A.V. Yurkovskaya, X.J. Wang, Magnetic field effect in natural cryptochrome explored with model compound, Sci. Rep. 7 (2017).

[63] T.M. Zollitsch, L.E. Jarocha, C. Bialas, K.B. Henbest, G. Kodali, P.L. Dutton, C.C. Moser, C.R. Timmel, P.J. Hore, S.R. Mackenzie, Magnetically sensitive radical photochemistry of non-natural flavoproteins, J. Am. Chem. Soc. 140 (2018) 8705-8713.

[64] R. Haberkorn, Density matrix description of spin-selective radical pair reactions, Mol. Phys. 32 (1976) 1491-1493.

[65] O. Efimova, P.J. Hore, Role of exchange and dipolar interactions in the radical pair model of the avian magnetic compass, Biophys. J. 94 (2008) 1565-1574.

[66] P.J. Hore, Upper bound on the biological effects of $50 / 60 \mathrm{~Hz}$ magnetic fields mediated by radical pairs, Elife 8 (2019).

[67] A. Peres, Separability criterion for density matrices, Phys. Rev. Lett. 77 (1996) 1413-1415.

[68] M. Horodecki, P. Horodecki, R. Horodecki, Separability of mixed states: Necessary and sufficient conditions, Phys. Lett. A 223 (1996) 1-8.

[69] G. Vidal, R.F. Werner, Computable measure of entanglement, Phys. Rev. A 65 (2002) 032314.

[70] H.J. Hogben, T. Biskup, P.J. Hore, Entanglement and sources of magnetic anisotropy in radical pair-based avian magnetoreceptors, Phys. Rev. Lett. 109 (2012).

[71] T. Baumgratz, M. Cramer, M.B. Plenio, Quantifying Coherence, Phys. Rev. Lett. 113 (2014).

[72] T. Möller, B. Trumbore, Fast, minimum storage ray-triangle intersection, Journal of Graphics Tools 2 (2012) 21-28.

[73] M.J. Frisch, G.W. Trucks, H.B. Schlegel, G.E. Scuseria, M.A. Robb, J.R. Cheeseman, G. Scalmani, V.G. Barone, A. Petersson, H. Nakatsuji, X. Li, M. Caricato, A. Marenich, J. Bloino, B. Janesko, G.R. Gomperts, B. Mennucci, H.P. Hratchian, J.V. Ortiz, A.F. Izmaylov, J.L. Sonnenberg, D. Williams-Young, F. Ding, F. Lipparini, F. Egidi, J. Goings, B. Peng, A. Petrone, T. Henderson, D. Ranasinghe, V.G. Zakrzewski, J. Gao, N. Rega, G. Zheng, W. Liang, M. Hada, M. Ehara, K. Toyota, R. Fukuda, J. Hasegawa, M. Ishida, T. Nakajima, Y. Honda, O. Kitao, H. Nakai, T. Vreven, K. Throssell, J.A. Montgomery, Jr., J.E. Peralta, F. Ogliaro, M. Bearpark, J.J. Heyd, E. Brothers, K.N. Kudin, V.N. Staroverov, T. Keith, R. Kobayashi, J. Normand, K. Raghavachari, A. Rendell, J.C. Burant, S.S. Iyengar, J. Tomasi, M. Cossi, J.M. Millam, M. Klene, C. Adamo, R. Cammi, J.W. Ochterski, R.L. Martin, K. 
Morokuma, O. Farkas, J.B. Foresman, D.J. Fox, Gaussian 09, Revision D.01, Gaussian, Inc., Wallingford CT, 2013.

[74] N.M. Atherton, Principles of electron spin resonance, Ellis Horwood, Chichester, 1993.

[75] D.R. Kattnig, C. Nielsen, I.A. Solov'yov, Molecular dynamics simulations disclose early stages of the photo-activation of cryptochrome 4, New J. Phys. 20 (2018).

[76] A. Günther, A. Einwich, E. Sjulstok, R. Feederle, P. Bolte, K.W. Koch, I.A. Solov'yov, H. Mouritsen, Double-Cone Localization and Seasonal Expression Pattern Suggest a Role in Magnetoreception for European Robin Cryptochrome 4, Curr. Biol. 28 (2018) 211-223.

[77] S. Franz, E. Ignatz, S. Wenzel, H. Zielosko, E.P.G.N. Putu, M. Maestre-Reyna, M.D. Tsai, J. Yamamoto, M. Mittag, L.O. Essen, Structure of the bifunctional cryptochrome aCRY from Chlamydomonas reinhardtii, Nucleic Acids Res. 46 (2018) 8010-8022.

[78] S. Richert, A. Rosspeintner, S. Landgraf, G. Grampp, E. Vauthey, D.R. Kattnig, Time-resolved magnetic field effects distinguish loose ion pairs from exciplexes, J. Am. Chem. Soc. 135 (2013) 15144-15152.

[79] G. Jeschke, B.C. Anger, B.E. Bode, J. Matysik, Theory of solid-state photo-CIDNP in the Earth's magnetic field, J. Phys. Chem. A 115 (2011) 9919-9928.

[80] S.S. Thamarath, J. Heberle, P.J. Hore, T. Kottke, J. Matysik, Solid-state photo-CIDNP effect observed in phototropin LOV1-C57S by C-13 magic-angle spinning NMR spectroscopy, J. Am. Chem. Soc. 132 (2010) 15542-15543.

[81] A.M. Lewis, D.E. Manolopoulos, P.J. Hore, Asymmetric recombination and electron spin relaxation in the semiclassical theory of radical pair reactions, J. Chem. Phys. 141 (2014).

[82] D.E. Manolopoulos, P.J. Hore, An improved semiclassical theory of radical pair recombination reactions, J. Chem. Phys. 139 (2013).

[83] F. Cailliez, P. Müller, T. Firmino, P. Pernot, A. de la Lande, Energetics of photoinduced charge migration within the tryptophan tetrad of an animal (6-4) photolyase, J. Am. Chem. Soc. 138 (2016) 1904-1915.

[84] P. Müller, J. Yamamoto, R. Martin, S. Iwai, K. Brettel, Discovery and functional analysis of a 4th electron-transferring tryptophan conserved exclusively in animal cryptochromes and (6-4) photolyases, Chem. Commun. 51 (2015) 15502-15505.

[85] N.F. Polizzi, A. Migliore, M.J. Therien, D.N. Beratan, Defusing redox bombs?, Proc. Natl. Acad. Sci. U. S. A. 112 (2015) 10821-10822.

[86] D.N. Beratan, S.S. Skourtis, I.A. Balabin, A. Balaeff, S. Keinan, R. Venkatramani, D.Q. Xiao, Steering Electrons on Moving Pathways, Acc. Chem. Res. 42 (2009) 1669-1678.

[87] M. Liedvogel, K. Maeda, K. Henbest, E. Schleicher, T. Simon, C.R. Timmel, P.J. Hore, H. Mouritsen, Chemical magnetoreception: Bird cryptochrome 1a is excited by blue light and forms long-lived radical-pairs, PLoS One 2 (2007) e1106.

[88] G. Baldissera, M. Byrdin, M. Ahmad, K. Brettel, Light-induced electron transfer in a cryptochrome blue-light photoreceptor, Nat. Struct. Biol. 10 (2003) 489-490.

[89] J.M. Cai, G.G. Guerreschi, H.J. Briegel, Quantum control and entanglement in a chemical compass, Phys. Rev. Lett. 104 (2010) 220502.

[90] I.K. Kominis, Magnetic sensitivity and entanglement dynamics of the chemical compass, Chem. Phys. Lett. 542 (2012) 143-146.

[91] Y.T. Zhang, G.P. Berman, S. Kais, Sensitivity and entanglement in the avian chemical compass, Phys. Rev. E 90 (2014).

[92] R.H. Keens, S. Bedkihal, D.R. Kattnig, Magnetosensitivity in dipolarly coupled three-spin systems, Phys. Rev. Lett. 121 (2018) 096001.

[93] J.J. Sakurai, J. Napolitano, Modern quantum mechanics, 2nd ed., Addison-Wesley, Boston, 2011. 


\section{Figures and Tables}

Table 1: Relative and absolute anisotropies of the singlet yield for radical pairs comprising the flavin anion radical and either the tryptophan cation radical or the tyrosine radical at different relative orientations and a radical pair lifetime of $1 \mu \mathrm{s}$.

\begin{tabular}{|c|c|c|c|c|c|c|c|}
\hline & $\mathrm{B}^{\bullet}$ & $B_{0}$ & $\alpha /^{\circ}$ & $\beta /{ }^{\circ}$ & $\gamma /{ }^{\circ}$ & $\Gamma_{\mathrm{s}} / \%$ & $\Delta_{\mathrm{s}} / 10^{5}$ \\
\hline \multirow{4}{*}{$\begin{array}{l}\text { maximal } \\
\text { relative } \\
\text { anisotropy }\end{array}$} & $\mathrm{W}$ & $50 \mu \mathrm{T}$ & 86 & 5 & 107 & 0.271 & 73.8 \\
\hline & & $1 \mathrm{mT}$ & 116 & 88 & -84 & 9.89 & 2910 \\
\hline & & $5 \mathrm{mT}$ & 54 & 179 & -144 & 4.19 & 1970 \\
\hline & $Y$ & $50 \mu \mathrm{T}$ & 179 & 88 & 174 & 0.963 & 251 \\
\hline \multirow{2}{*}{$\begin{array}{l}\text { maximal } \\
\text { optimality }\end{array}$} & W & $50 \mu \mathrm{T}$ & -27 & 93 & 0 & 0.099 & 2.52 \\
\hline & $Y$ & $50 \mu \mathrm{T}$ & 144 & 95 & 4 & 0.802 & 210 \\
\hline \multirow[t]{6}{*}{ DmCry } & $W_{A}=W 420$ & $50 \mu \mathrm{T}$ & -115 & 80 & 90 & 0.110 & 2.80 \\
\hline & $W_{B}=W 397$ & $50 \mu \mathrm{T}$ & 82 & 102 & 99 & 0.105 & 2.69 \\
\hline & $W_{C}=W 342$ & $50 \mu \mathrm{T}$ & 136 & 141 & 13 & 0.183 & 4.87 \\
\hline & $W_{D}=W 394$ & $50 \mu \mathrm{T}$ & 70 & 83 & 9 & 0.100 & 2.56 \\
\hline & $W_{C}=W 342$ & $1 \mathrm{mT}$ & 136 & 141 & 13 & 8.53 & 2530 \\
\hline & $W_{C}=W 342$ & $5 \mathrm{mT}$ & 136 & 141 & 13 & 4.06 & 1910 \\
\hline AtCry1 & $W_{C}=W 324$ & $50 \mu \mathrm{T}$ & 123 & 112 & 1 & 0.112 & 2.87 \\
\hline \multirow[t]{2}{*}{ ErCry4 } & $W_{c}=W 318$ & $50 \mu \mathrm{T}$ & 126 & 130 & -7 & 0.149 & 3.90 \\
\hline & $W_{D}=W 369$ & $50 \mu \mathrm{T}$ & 78 & 97 & -11 & 0.103 & 2.62 \\
\hline
\end{tabular}




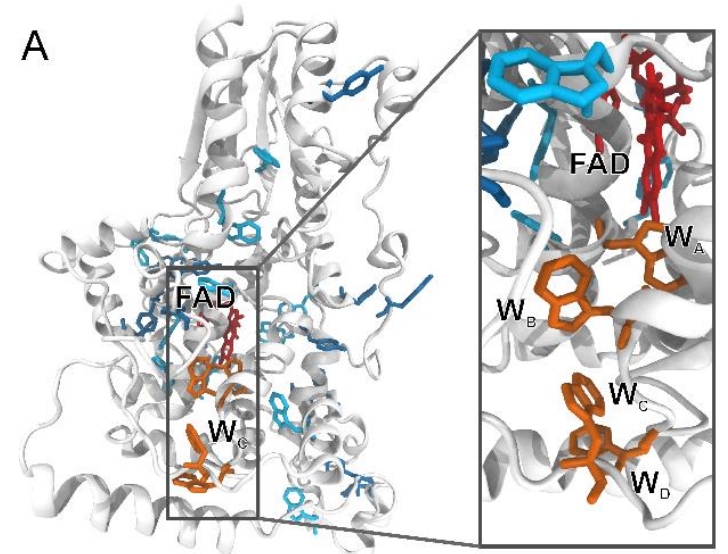

B

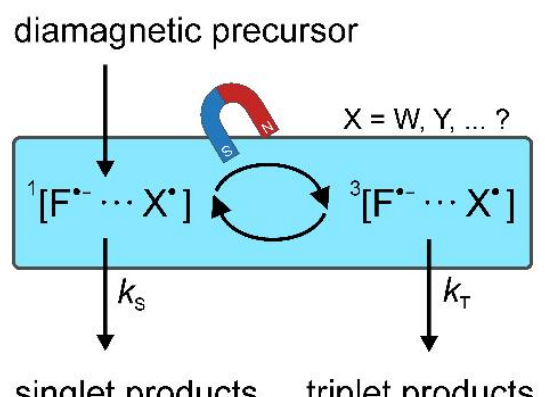

Figure 1: A) Structure of the cryptochrome from D. melanogaster (PDB ID: 4GU5) with potentially electroactive tyrosine (blue) and tryptophan (cyan) residues highlighted. The tryptophan tetrad, which comprises four tryptophan residues and forms a highly conserved electron transfer pathway, is shown in orange. The flavin cofactor (flavin adenine dinucleotide; FAD) is drawn in red. B) Schematic illustration of the origin of the origin of the magnetosensitivity: coherent singlet-triplet conversion in the radical pair in combination with spin-selective reactivity. $\mathrm{F}^{\circ-}$ stands for the radical anion of the flavin cofactor; $X^{\bullet}$ denotes a radical partner, whose identity is subject to ongoing controversy. In in vitro experiments, $X^{\bullet}$ was found to correspond to a tryptophan radical from the tryptophan triad/tetrad, $W_{c^{\circ}}$. In vivo, alternative structures and electron transfer pathways could give rise to different radical pairs. 
A
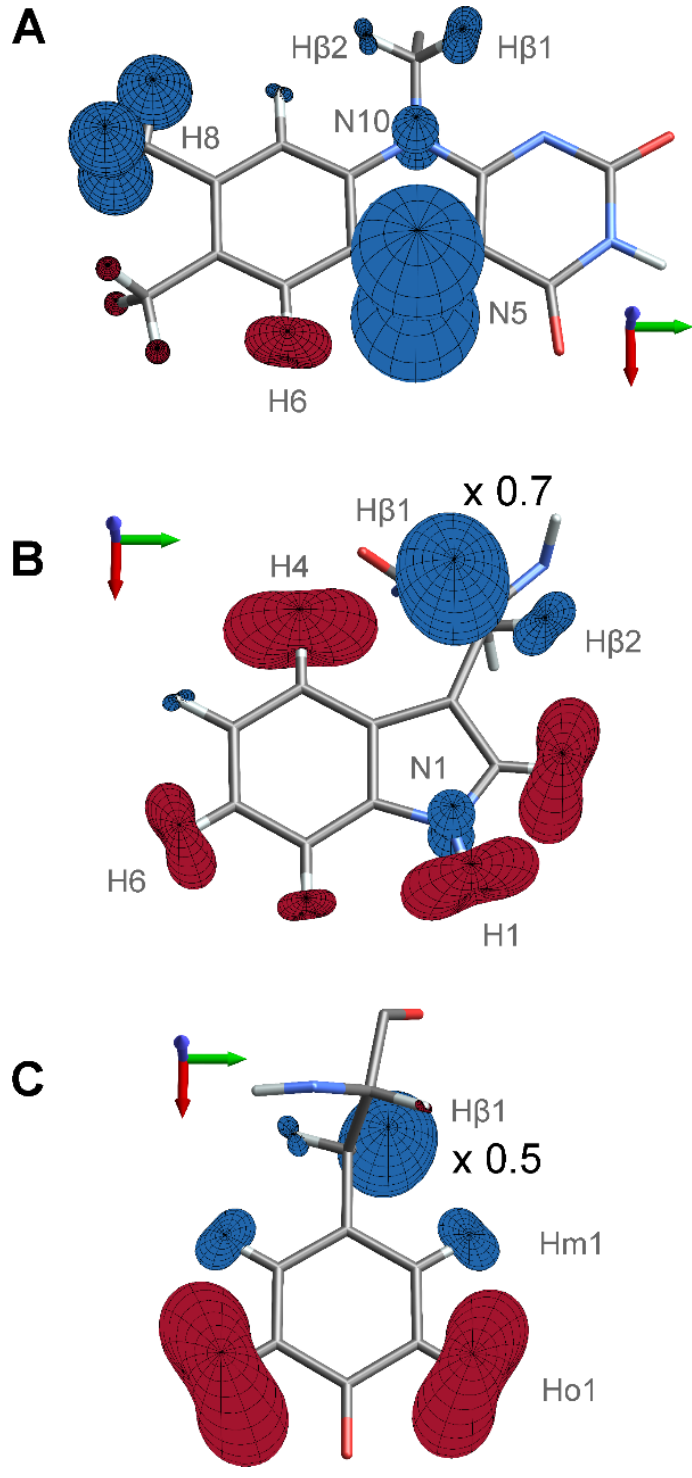

Figure 2: Representation of the considered hyperfine interactions in A) $\mathrm{F}^{\bullet-}$ (12 coupled nuclei), B) $\mathrm{W}^{\bullet+}$ (9 coupled nuclei) and C) $Y^{\bullet}(7$ coupled nuclei) superimposed on the (truncated) structures of the radicals. Here, the hyperfine contours are drawn such that $1 \AA$ corresponds to $17 \mathrm{MHz}$. For $\mathrm{W}^{\bullet+}$ and $\mathrm{Y}^{\bullet}$ a hyperfine interaction of a $\beta$-proton was scaled down by the indicated factor. The local axes systems are shown. Details of their definition in relation to the molecular structure are provided in the Supporting Information (Figure S4). The hyperfine interaction tensors, which are listed in the Supporting Information, Tables S1 to S3, were calculated at the UB3LYP/EPR-III level of theory. 


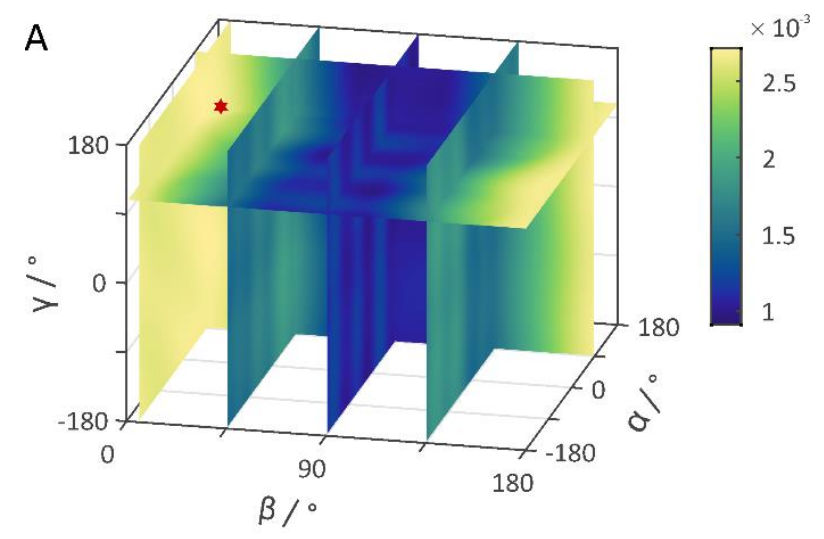

B

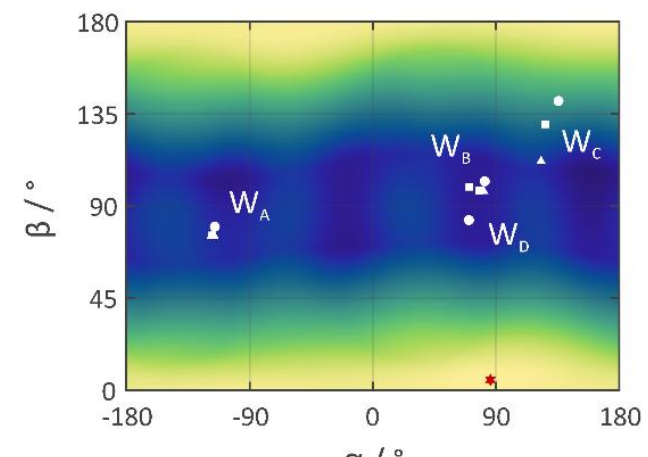

$\alpha /^{\circ}$

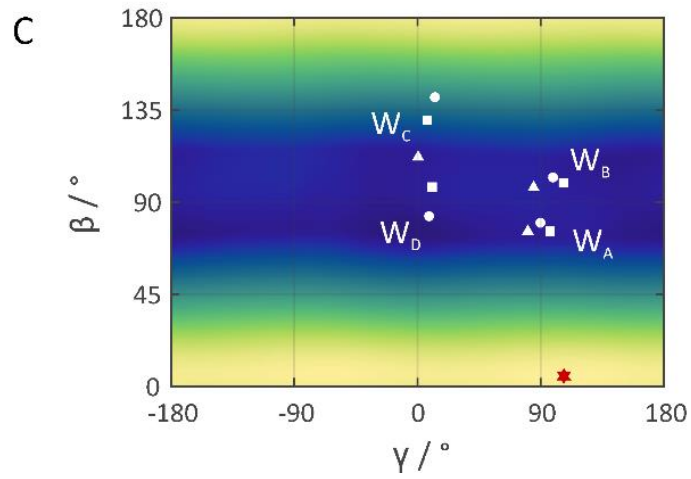

Figure 3: Relative anisotropy of the singlet yield of $\left[\mathrm{F}^{\cdot-} \mathrm{W}^{\bullet+}\right]$ as a function of the relative orientation of the radicals. The magnetic field intensity was $B_{0}=50 \mu \mathrm{T}$ and the radical pair lifetime $1 \mu \mathrm{s}$. A) gives selected slices through the orientational dependent yield as a function of the three Euler angles, $\alpha, \beta$, $\gamma$ that specify the orientation of the flavin radical in the molecular frame of $\mathrm{W}^{\circ+}$. B) and C) provide two dimensional representations for the $\alpha, \beta$-plane and $\beta, \gamma$-plane passing through the orientation of maximal anisotropy ( $\alpha=86^{\circ}, \beta=5^{\circ}, \gamma=107^{\circ}$; marked by red star), respectively. The (projections of the) relative orientations of tryptophans of the tryptophan triad/tetrad have been indicated for DmCry (bullets), AtCry1 (triangles) and ErCry4 (squares). The same color bar applies to all panels. 

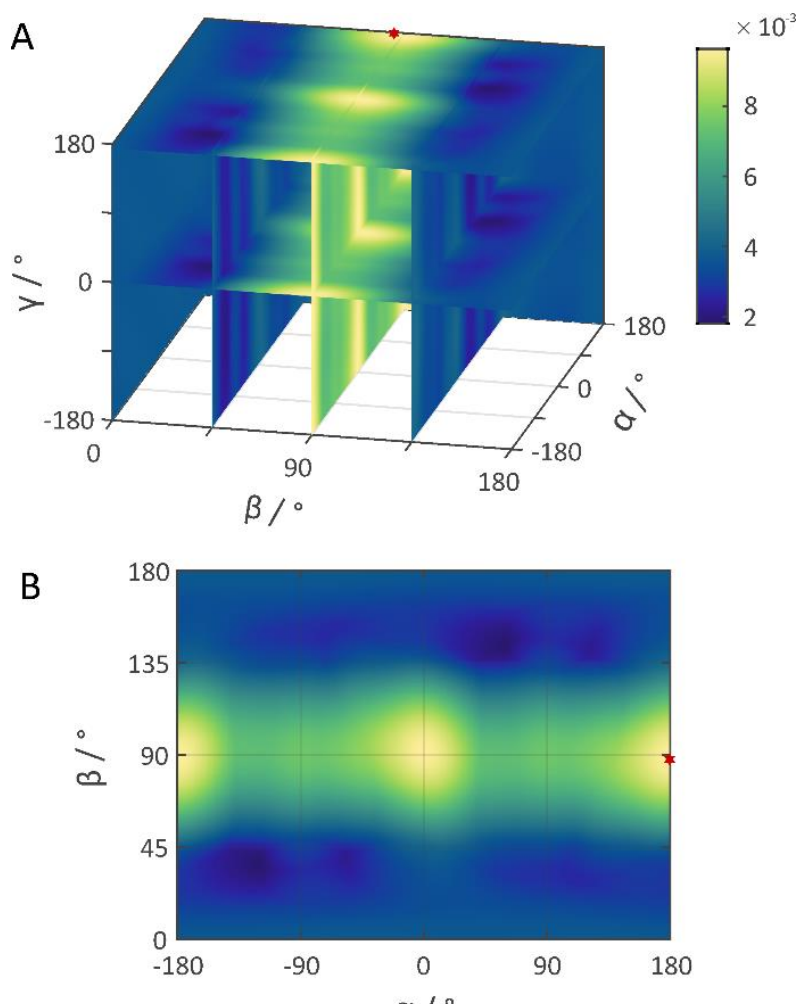

$\alpha /{ }^{\circ}$

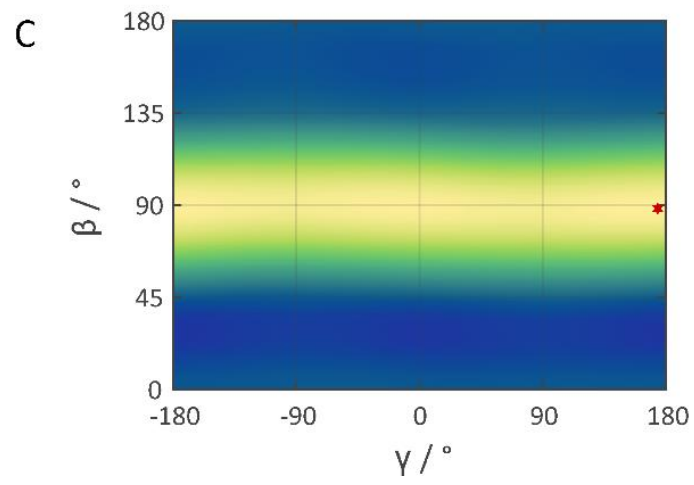

Figure 4: Dependence of the relative singlet yield anisotropy of $\left[\mathrm{F}^{{ }^{--}} \mathrm{Y}^{*}\right]$ in a magnetic field of $B_{0}=50$ $\mu \mathrm{T}$ as a function of the relative orientation of the radicals. A) gives selected slices through the orientational dependent yield as a function of the three Euler angles, $\alpha, \beta, \gamma$ that specify the orientation of the flavin radical in the molecular frame of $\mathrm{Y}^{\bullet}$. B) and $\mathrm{C}$ ) provide two dimensional representations for the $\alpha, \beta$-plane and $\beta, \gamma$-plane passing through the orientation of maximal anisotropy ( $\alpha=179^{\circ}, \beta=88^{\circ}, \gamma=174^{\circ}$; marked by red star), respectively. The radical pair lifetime was $1 \mu \mathrm{s}$. 

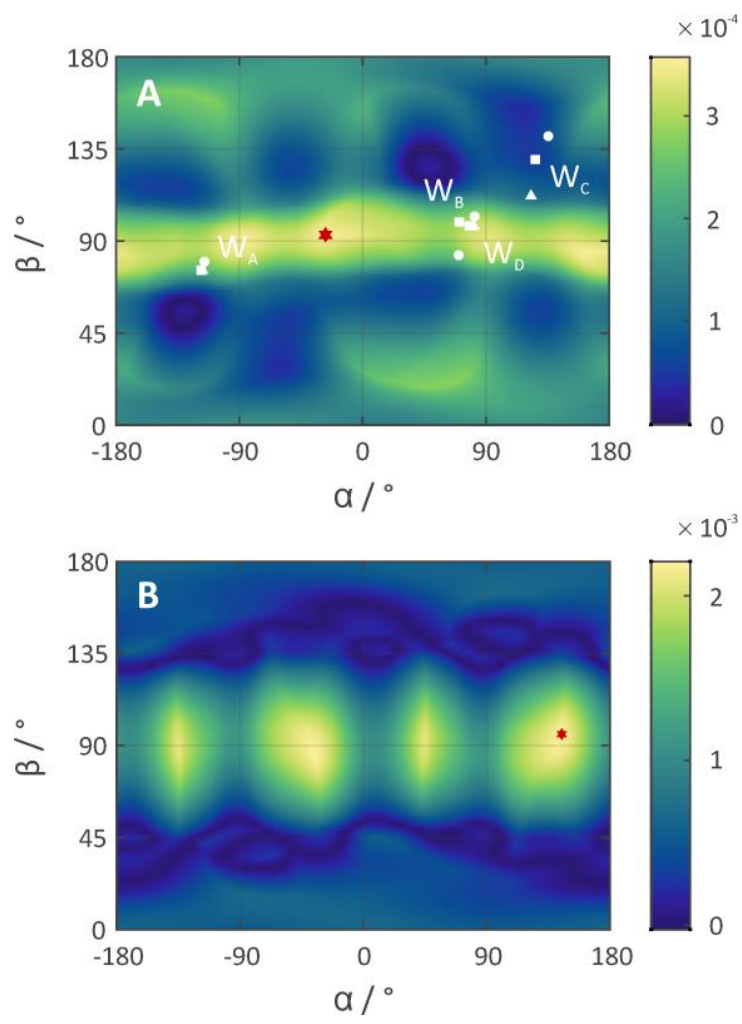

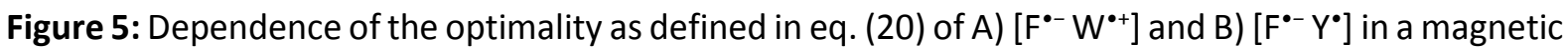
field of $B_{0}=50 \mu \mathrm{T}$ as a function of the relative orientation of the radicals. The $\alpha, \beta$-dependence is shown for the $\gamma$ of maximal optimality. For $\left[\mathrm{F}^{\circ-} \mathrm{W}^{\bullet+}\right]$ and $\left[\mathrm{F}^{{ }^{--}} \mathrm{Y}^{\circ}\right]$, maximal optimality is realized for $\alpha=$ $-27^{\circ}, \beta=93^{\circ}, \gamma=0^{\circ}$ and for $\alpha=144^{\circ}, \beta=95^{\circ}, \gamma=4^{\circ}$, respectively. The orientation of maximal optimality is marked by a red star. For $\left[\mathrm{F}^{\circ-} \mathrm{W}^{\circ+}\right](\mathrm{A})$ the tryptophans of the tryptophan triad/tetrad have been indicated for DmCry (bullets), AtCry1 (triangles) and ErCry4 (squares). The radical pair lifetime was $1 \mu \mathrm{s}$. 